\title{
Limits on Reddening and Gas-to-Dust Ratios for Seven Intermediate Redshift Damped Ly $\alpha$ Absorbers from Diffuse Interstellar Bands
}

\author{
Brandon Lawton ${ }^{1}$, Christopher W. Churchill ${ }^{1}$, Brian A. York ${ }^{2}$, Sara L. Ellison $^{2}$, \\ Theodore P. Snow ${ }^{3}$, Rachel A. Johnson ${ }^{4}$, Sean G. Ryan ${ }^{5}$, And Chris R. Benn ${ }^{6}$
}

\begin{abstract}
We present equivalent width measurements and limits of six diffuse interstellar bands (DIBs, $\lambda 4428, \lambda 5705, \lambda 5780, \lambda 5797, \lambda 6284$, and $\lambda 6613$ ) in seven damped Ly $\alpha$ absorbers (DLAs) over the redshift range $0.091 \leq z \leq 0.524$, sampling $20.3 \leq \log N(\mathrm{HI}) \leq 21.7$. DIBs were detected in only one of the seven DLAs, that which has the highest reddening and metallicity. Based upon the Galactic DIB- $N(\mathrm{HI})$ relation, the $\lambda 6284$ DIB equivalent width upper limits in four of the seven DLAs are a factor of 4-10 times below the $\lambda 6284$ DIB equivalent widths observed in the Milky Way, but are not inconsistent with those present in the Magellanic Clouds. Assuming the Galactic DIB- $E(B-V)$ relation, we determine reddening upper limits for the DLAs in our sample. Based upon the $E(B-V)$ limits, the gas-to-dust ratios, $N(\mathrm{HI}) / E(B-V)$, of the four aforementioned DLAs are at least $\sim 5$ times higher than that of the Milky Way ISM. The ratios of two other DLAs are at least a factor of a few times higher. The best constraints on reddening derive from the upper limits for the $\lambda 5780$ and $\lambda 6284$ DIBs, which yield $E(B-V) \leq 0.08$ for four of the seven DLAs. Our results suggest that, in DLAs, quantities related to dust, such as reddening and metallicity, appear to have a greater impact on DIB strengths than does Hi gas abundance; the organic molecules likely responsible for DIBs in DLA selected sightlines are underabundant relative to sightlines in the Galaxy of similarly high $N(\mathrm{HI})$. With regards to the study of astrobiology, this could have implications for the abundance of organic molecules in redshifted galaxies. However, since DLAs are observed to have low reddening, selection bias likely plays a role in the apparent underabundance of DIBs in DLAs.
\end{abstract}

\footnotetext{
${ }^{1}$ Department of Astronomy, MSC 4500, New Mexico State University, P.O. Box 30001, Las Cruces, NM 88003; blawton@nmsu.edu,cwc@nmsu.edu

${ }^{2}$ Department of Physics \& Astronomy, University of Victoria, 3800 Finnerty Rd., Victoria, V8W 1A1, British Columbia, Canada; briany@uvic.ca, sarae@uvic.ca

${ }^{2}$ Center for Astrophysics and Space Astronomy, University of Colorado at Boulder, 389 UCB, Boulder, CO 80309; Theodore.Snow@colorado.edu

${ }^{4}$ Oxford Astrophysics, Denys Wilkinson Building, Keble Road, Oxford OX1 3RH, UK; raj@astro.ox.ac.uk

${ }^{5}$ Centre for Astrophysics Research, University of Hertfordshire, College Lane, Hatfield AL10 9AB, UK; s.g.ryan@herts.ac.uk

${ }^{6}$ Isaac Newton Group, Apartado 321, E-38700 Santa Cruz de La Palma, Spain; crb@ing.iac.es
} 
Subject headings: dust, extinction - galaxies: ISM and abundances - quasars: absorption lines and individual (AO 0235+164, Q0738+313, B2 0827+243, PKS 0952+179, PKS 1127-145, Q1229-020) - techniques: spectroscopic

\section{Introduction}

Since their discovery by Heger (1922), several hundred diffuse interstellar bands (DIBs) have been studied (Galazutdinov et al. 2000; Jenniskens \& Desert 1994; Tuairisg et al. 2000; Weselak et al. 2000; Hobbs et al. 2007), and yet no positive identifications of the carriers have been made. The DIBs span the visible spectrum between 4000 and 13,000 $\AA$. Despite no positive identifications, several likely organic molecular candidates have emerged as the sources of the DIBs, including polycyclic aromatic hydrocarbons (PAHs), fullerenes, long carbon chains, and polycyclic aromatic nitrogen heterocycles (PANHs) (e.g., Herbig 1995; Snow 2001; Cox \& Spaans 2006a; Hudgins et al. 2005). The organic-molecular origin of the DIBs may give them an importance to astrobiology; PAHs are now considered an important early constituent to the inventory of organic compounds on Earth (Bada \& Lazcano 2002). Via their infrared emitting vibrational bands, PAHs have been observed in high redshift dusty ultra-luminous infrared galaxies (ULIRGs, e.g., Yan et al. 2005). Searching for DIBs using the technique of quasar absorption lines provides a different approach for charting the presence of possible organics to high redshift. As such, observing DIBs in high redshift galaxies may offer an independent method for constraining the environmental conditions in early-epoch galaxies governing the abundances of organic molecules, determining the cosmic epoch at which organic molecules first formed, and ultimately charting their evolution with redshift.

Aside from the hundreds of detections within the Galaxy (e.g. Galazutdinov et al. 2000; Jenniskens \& Desert 1994; Tuairisg et al. 2000; Weselak et al. 2000; Hobbs et al. 2007), DIBs have been detected in the Magellanic Clouds (Weltv et al. 2006; Cox et al. 2006b, 2007), M31 (Cordiner et al. 2008), seven starburst galaxies (Heckman \& Lehnert 2000), active galaxy Centaurus A via supernova 1986A (Rich 1987), spiral galaxy NGC 1448 via supernovae 2001el and 2003hn (Sollerman et al. 2005), one damped Ly $\alpha$ absorber (DLA) at $z=0.524$ toward the quasar (QSO) AO $0235+164$ (Junkkarinen et al. 2004; York et al. 2006), and one $z=0.157$ CaII selected absorber toward QSO J0013-0024 (Ellison et al. 2008).

There are several environmental factors, such as Hi column density (Herbig 1995; Welty et al. 2006), reddening (Welty et al. 2006), and metallicity and ionizing radiation (Cox et al. 2007), that are related to DIB strengths. In the Galaxy, DIB absorption strengths correlate strongly with $N(\mathrm{HI})$ (Herbig 1995; Welty et al. 2006). However, in the Magellanic Clouds, DIBs are weaker by factors of 7-9 (LMC) and 20 (SMC) compared to those observed in the Galaxy with similar N(Hi) (Welty et al. 2006). It is not known whether other galaxies in the Local Group and beyond obey the Galactic DIB- $N(\mathrm{HI})$ relation, or, like the Magellanic Clouds, show departures from this relation. Galaxies with high $N(\mathrm{HI})$ observed in absorption (i.e., DLAs) that reside at low to intermediate redshifts (where the prominent DIBs fall in the optical region) provide excellent astrophysical 
laboratories with which to investigate this issue.

In this paper we search for $\lambda 4428, \lambda 5780, \lambda 5797, \lambda 6284$, and $\lambda 6613$ DIB absorption in seven low to intermediate redshift DLAs. In $\S 2$, we give a brief summary of each intervening DLA in our sample. In $\S$ 3, we discuss the spectroscopic observations and data reduction of the background QSOs. In $\S$ 4, we explain the procedure of our analysis, and the resulting spectra. In $\S 5$, we present our results and compare our data to the Galactic DIB- $N$ (HI) relation, deduce upper limits for the reddening, $E(B-V)$, determine lower limits on the gas-to-dust ratios, and discuss the role of metallicity for our sample of DLAs. We conclude in $\S 6$.

\section{DLA Sample}

To potentially maximize our chances of detecting DIBs, and to test the Galactic DIB- $N(\mathrm{HI})$ relation in redshifted galaxies, we selected DLAs toward background QSOs having the highest $N(\mathrm{HI})$ in absorption. We limited the redshift range such that the strongest DIBs would fall in the optical region. Thus, we selected the highest $N(\mathrm{HI})$ DLA galaxies in the redshift range $0.09<$ $z<0.52$. DLAs, by definition, have a large neutral gas column density $\left(N(\mathrm{HI}) \geq 2 \times 10^{20} \mathrm{~cm}^{-2}\right)$. However, they are observed to have low reddening $(E(B-V)<0.04)$ (Ellison et al. 2005) and low metallicity (typically $Z \sim 0.1 Z_{\odot}$ ). The low metallicity and reddening of DLAs suggest that the gaseous environments selected by DLA absorption differ from the Galactic ISM in which DIBs are observed. As we discuss in $\S 5.2$, the low reddening of DLAs is not merely a bias arising from the necessity of low extinction if the background quasar is to be detected at all, since DLAs in radio-selected quasars have similarly low reddenings.

Below, we describe the basic properties of each of the seven DLAs comprising our sample. Table 1 summarizes the main parameters, including abundance information. The columns, from left to right, list the QSO, absorption redshift of the DLA, column density of neutral hydrogen, the zinc abundance, the iron abundance, and the associated references. The Ly $\alpha$ line is used to measure the DLA redshift. All impact parameters have been converted to a $\Lambda$ CDM cosmology $\left(H_{0}=70 \mathrm{~km} \mathrm{~s}^{-1} \mathrm{Mpc}^{-1}, \Omega_{m}=0.3, \Omega_{\Lambda}=0.7\right)$.

The $z_{a b s}=0.524$ DLA $(\# 1)$ toward AO $0235+164$ is unique in our sample for many reasons. It is the only DLA known to have DIB absorption 1, the $\lambda 4428$ DIB (Junkkarinen et al. 2004) and the $\lambda 5705$ and $\lambda 5780$ DIBs (York et al. 2006). The $\lambda 5797, \lambda 6284$, and $\lambda 6613$ DIBs have limits that are not as sensitive due to sky lines. The associated optical galaxy with the smallest impact parameter, $6.7 h_{70}^{-1} \mathrm{kpc}$, is a late-type spiral and is assumed to be the absorber (Rao et al. 2003; Burbidge et al. 1996; Yanny et al. 1989). However, Chen \& Lanzetta (2003) argue that many of the galaxies in the optical field have the same redshift and may collectively be responsible for the

\footnotetext{
${ }^{1}$ The DIB-bearing CaII absorber reported by Ellison et al. (2008) is likely to be a DLA. However, this cannot be confirmed without a spectrum of the Ly $\alpha$ absorption.
} 
DLA absorption. The adopted neutral hydrogen column density is from Junkkarinen et al. (2004) and is consistent with $N(\mathrm{HI})=(4.5 \pm 0.4) \times 10^{21} \mathrm{~cm}^{-2}$ from Turnshek et al. (2003). The measured metallicities (Turnshek et al. 2003; Junkkarinen et al. 2004) assume solar abundance ratios and solar metallicity for the foreground Galactic absorption. Junkkarinen et al. (2004) state that the difference between their metallicities and those of Turnshek et al. (2003) are likely due to different realizations of the noise in the X-ray data and/or the variability (Rieke et al. 1976) of this QSO. Despite the differences in these measurements, this DLA has the highest metallicity in our sample. The DLA also has significant reddening with $E(B-V)=0.23, R_{v}=2.5$, and a strong graphitic dust feature at $2175 \AA$.

The $z=0.091$ DLA (\#2) toward Q0738+313 (OI363) is probably a low surface brightness galaxy with an impact parameter of $<3.5 h_{70}^{-1} \mathrm{kpc}$ (Turnshek et al. 2001). This DLA is one of two found along the QSO sightline of Q0738+313 and was first reported by Rao \& Turnshek (1998).

The other DLA, $z_{a b s}=0.221(\# 3)$, toward the QSO Q0738+313 is probably a dwarf spiral with an impact parameter of $20.5 h_{70}^{-1} \mathrm{kpc}$ (Turnshek et al. 2001).

The $z_{a b s}=0.518$ DLA (\#4) toward B2 $0827+243$ is likely a disturbed spiral galaxy with extended gas that produces the observed hydrogen absorption at an impact parameter of $38.2 h_{70}^{-1} \mathrm{kpc}$ (Rao et al. 2003). Khare et al. (2004) note that it appears the DLA requires a significant radiation field, similar to the radiation within the dense clouds in our Galaxy, to create a low $N(\mathrm{Fe} \mathrm{I}) / N(\mathrm{Fe}$ II) upper limit of $10^{-3}$.

The absorbing galaxy giving rise to the $z_{a b s}=0.239$ DLA $(\# 5)$ toward PKS 0952+179 has not been confirmed. However, two candidates lie within $<4.6 h_{70}^{-1} \mathrm{kpc}$ (Rao et al. 2003). The authors note that these two galaxies appear to be nearly edge-on and are classified as dwarf low surface brightness galaxies. The DLA appears to have multiple Ly $\alpha$ components around the central line at $z=0.239$ (Rao \& Turnshek 2000). The adopted hydrogen column density is measured from the central Ly $\alpha$ feature. However, the velocity structure suggests a possible clustering.

The $z_{a b s}=0.313$ DLA (\#6) toward PKS 1127-145 is possibly the remnant of a dwarf low surface brightness galaxy tidally disturbed by more massive spiral galaxies in the same field (Rao et al. 2003). The absorber appears to overlap the QSO point spread function, thus, Rao et al. (2003) find an upper limit for the impact parameter of $6.9 h_{70}^{-1} \mathrm{kpc}$. Turnshek et al. (2003) discuss the difficulty of determining a metallicity for this system due to uncertainties in their X-ray spectrum. We have estimated a lower limit of the iron abundance from a VLT/UVES spectrum kindly donated for this work by Dr. M. Murphy. The system has a complicated velocity structure. However, fixing the Doppler parameter, redshift, and number of Voigt profiles yields a lower limit on the column density of $\log N(\mathrm{Fe} I \mathrm{I})>15.7$ atoms $\mathrm{cm}^{-2}$. Repeating this analysis using the apparent optical depth (Savage \& Sembach 1991) gives a slightly more conservative limit of $\log N$ (FeII) $>15.2$ atoms cm $^{-2}$, which we adopt for the column density of iron. Assuming the Lodders (2003) solar abundances, we deduce a lower limit of $[\mathrm{Fe} / \mathrm{H}]>-2$.

The absorbing galaxy responsible for the $z_{a b s}=0.395$ DLA $(\# 7)$ along the Q1229-029 sight- 
line is a low surface brightness galaxy with an impact parameter of $8.2 h_{70}^{-1} \mathrm{kpc}$ (Le Brun et al. 1997; Steidel et al. 1994).

\section{Observations and Data Reduction}

Observations of the seven DLAs were obtained with seven facilities toward six QSO sightlines between July 2002 and October 2005. The S/N of the QSO spectra range from 5-150 pixel ${ }^{-1}$. All quoted $\mathrm{S} / \mathrm{N}$ measurements are calculated in the regions of the expected locations of the redshifted $\lambda 4428, \lambda 5780, \lambda 5797, \lambda 6284$, and $\lambda 6613$ DIBs. The journal of observations is presented in Table 2 . Cataloged are the QSO, facility and instrument used in the observation, the grating/grism, the slit width, the UT date of the observation, the total exposure time in seconds, and the wavelength coverage of each spectrum in angstroms.

\subsection{Observations}

QSO spectra covering the $z=0.524, z=0.239$, and $z=0.395$ DLAs along the sightlines AO 0235+164, PKS 0952+179, and Q1229-020 were obtained with the FORS2 spectrograph on the Very Large Telescope (VLT). All resolutions are obtained by measuring unresolved sky emission lines. Observations of A0 0235+164 and Q1229-020 use the same grating and the resolutions agree within reasonable uncertainties.

A QSO spectrum covering the $z=0.091$ and $z=0.221$ DLAs along the Q0738+313 sightline was acquired with the DIS spectrograph on the Apache Point Observatory (APO) $3.5 \mathrm{~m}$ telescope. DIS is configured with a dichroic that splits the light to a blue chip and a red chip at $\sim 5500 \AA$. The resolution is measured directly from unresolved sky emission lines.

A Keck/HIRES spectrum of B2 $0827+243$ covering the DLA at $z=0.518$ was kindly provided by Dr. W. Sargent. The resolution is measured by unresolved atmospheric absorption lines (we did not have access to sky emission line data for this object).

QSO spectra covering the $z=0.239$ and $z=0.395$ DLAs along the sightlines toward PKS $0952+179$ and Q1229-020 were obtained using the ISIS spectrograph on the $4 \mathrm{~m}$ William Herschel Telescope (WHT). The resolutions were measured directly from unresolved sky emission lines; the uncertainties are relatively large because of the low $\mathrm{S} / \mathrm{N}$ in these data.

A UVES/VLT spectrum covering the $z=0.313$ DLA toward PKS 1127-145 was kindly provided by Dr. M. Murphy. The resolution is taken from Dekker et al. (2000). We did not have access to sky data for this spectrum nor were there any unresolved atmospheric absorption lines, so we could not estimate the resolution directly.

A QSO spectrum covering the $z=0.313$ DLA along the sightline toward PKS 1127-145 was 
obtained with the GMOS spectrograph on the $8.1 \mathrm{~m}$ Gemini South telescope. The resolution is taken from the online Gemini Science Operations GMOS Instrument Manual. We had limited sky data, so we did not estimate the resolution directly. However, due to large equivalent width limits of the GMOS data, the effect of uncertainties in resolution should be minimal and not affect results in this paper (see Table 3).

\subsection{Data Reduction}

With the exception of the VLT/UVES and Gemini/GMOS spectra of PKS 1127-145, the data were reduced using standard IRAF2 packages. The IRAF reduction process involved bias subtraction, flat-fielding, spectrum extraction, and wavelength calibration using standard lamps. Once wavelength calibrated, each spectrum was continuum fit manually using sfit to achieve the lowest residuals in the regions of the DIBs where no detections are observed. Near telluric lines or problematic sky subtraction, the continuum was fit using regions bracketing these features. The flux, uncertainty, sky (when acquired), and continuum spectra are normalized and then the individual spectra are optimally combined (using an algorithm of our own design). For the Gemini/GMOSS spectrum, data reduction was performed using the IRAF Gemini tools in the gmos package. The IRAF task, telluric, was also used on the Gemini/GMOS-S spectrum (York et al. 2006). The UVES spectrum was reduced using the standard ESO pipeline and a custom code called the UVES Post-Pipeline Echelle Reduction (Uves POPLER, Murphy 2006).

\section{Data Analysis and Spectra}

\subsection{Analysis of DIBs}

A modified method of Schneider et al. (1993) was developed and used to search for the presence of $\lambda 4428, \lambda 5780, \lambda 5797, \lambda 6284$, and $\lambda 6613$ DIB absorption. The Schneider et al. technique is optimized for objectively locating unresolved features in spectra. However, some DIBs are resolved in our spectra. Thus, we modified the Schneider et al. (1993) method to be optimized for both unresolved and resolved features by combining the DIB's intrinsic full width at half maximum (FWHM) with the instrumental spread function (ISF). The procedure is outlined in Appendix A.1. The calculation used by Schneider et al. (1993) (given in Eq. A4) transforms to Eq. A11,

In addition, we replaced the normalized flux error of Schneider et al. (1993) by the residuals of the data in pixels where the normalized flux deviates significantly from the continuum (see Eqs. A8, A9, and A10). This results in a more conservative detection threshold (equivalent width

\footnotetext{
${ }^{2}$ IRAF is distributed by the National Optical Astronomy Observatories, which are operated by the Association of Universities for Research in Astronomy, Inc., under cooperative agreement with the National Science Foundation.
} 
limit) in the cases of problematic sky subtraction or continuum fits. As an example of this method, Fig. 1 1 a displays the relative flux of Q0738+313 in the region of the expected positions of the $\lambda 5780$ and $\lambda 5797$ DIBs for the $z=0.091$ DLA. Fig. 1b contains the sky data. Fig. 1 contains the associated $3 \sigma$ rest-frame detection thresholds for a Gaussian profile with the expected FWHM of the redshifted $\lambda 5780$ DIB. Both the $\lambda 5780$ and $\lambda 5797$ DIBs are unresolved. However, if they had been resolved they would have yielded different equivalent width limits because the limits depends upon the redshifted FWHM for resolved DIBs. The problematic sky subtraction at $6300 \AA$ and the problematic continuum fit at $6314 \AA$ result in conservative limits due to large residuals (see Eqs. A8, A10, and A11). The region around the $\lambda 5797$ DIB is "clean" in that no residuals are used in the equivalent width limit calculations. The equivalent width limits of the DIBs in the AO $0235+164$ DLA measured by York et al. (2006) are less stringent but not inconsistent with those measured by this method.

Possible detections were visually inspected to determine if they were a DIB or perhaps another feature or sky line residual. Checking each candidate feature is essential because of a small uncertainty in the rest wavelength of each DIB as well as a small uncertainty in the redshift of DIB absorbing gas relative to the Ly $\alpha$ determined redshift of the DLA. For most DIBs, we were able to measure only equivalent width limits.

To quantify confidence levels in the measured detection thresholds determined with our modified method, we have estimated the $1 \sigma$ uncertainties in the measured equivalent width limits. There is some uncertainty in the resolution (ISF) of each spectrum. We estimate the uncertainty in the resolution as the standard deviation of the $\lambda /$ FWHM ratios of unresolved sky absorption lines or emission lines, where the $\lambda$ are the line centers. If sky data are not available, we excluded the uncertainty in resolution. In addition, there is uncertainty in the accuracy of the continuum fits, which we estimated using the technique of Sembach et al. (1991). Of importance, is the fact that DIBs have measured uncertainties in their rest-frame wavelengths and FWHMs (Jenniskens \& Desert 1994). The uncertainty in the equivalent width limit due to uncertainty in the wavelength is the standard deviation of the individual equivalent width limits computed over the range of the redshifted DIB wavelength uncertainty. The full explanation of "error" propagation to obtain uncertainties in the equivalent width limits is given in Appendix A.2 (see Eq. A12).

Contained within Fig. 1 is an example of our equivalent width limit analysis including information on the effects of uncertainties in the wavelength of the band center. If a redshifted DIB wavelength is near a problematic region then an uncertainty in rest wavelength can introduce a large uncertainty in a measured equivalent width limit. This is a more substantial issue for the $\lambda 4428$ DIB, which has the largest uncertainty in its rest-frame wavelength $(\sim 1.4 \AA)$. For the majority of the equivalent width limits, the largest uncertainty is due to the continuum fit.

Only three DIBs have been detected in DLAs, the $\lambda 4428$ DIB reported in Junkkarinen et al. (2004), and the $\lambda 5705$ and $\lambda 5780$ DIBs reported in a companion paper of this work by York et al. (2006). All three arise in the $z=0.524$ DLA toward AO $0235+164$. The equivalent widths were 
calculated by summing the individual equivalent widths of each pixel. Both of the detections from York et al. (2006) were at least one resolution element away from sky lines. The undetected $\lambda 5797$ and $\lambda 6284$ DIBs toward the $z=0.524$ AO $0235+164$ DLA were located within strong sky lines. York et al. (2006) estimated the equivalent width limits for these DIBs using synthetic absorption features with varying FWHMs. The undetected $\lambda 6613$ DIB is near a sky line; York et al. (2006) estimated its equivalent width limit directly from the signal-to-noise, dispersion of the chip, and number of pixels.

\subsection{Measurements and Spectra}

The measured equivalent widths, equivalent width limits $(3 \sigma)$, and their $1 \sigma$ uncertainties are listed in Table 3. Tabulated are the DLA (by number), the corresponding QSO, the absorption redshift of the DLA, the facility and instrument, and the rest-frame equivalent widths or limits, with uncertainties $(\mathrm{m} \AA$ ) of each of the DIBs if they were observable. Spectra from which the most stringent limits were obtained are shown in Figs. 2 2 . Each panel $(a)-(e)$ displays the region around a redshifted DIB. The upper sub-panels display the normalized flux (histogram) with the expected positions of the DIBs (marked by ticks) based upon the Ly $\alpha$ redshift. With the exceptions of Figs. 5 and 7 , the center sub-panels display the uncertainty spectra of the associated normalized fluxes, and the lower sub-panels display the sky counts normalized by the continuum. Sky data were unavailable for B2 $0827+243$ at $z=0.518$ and PKS $1127-145$ at $z=0.313$.

The smooth thin curves through the data are the expected observed DIB absorption profiles based on the measured $N(\mathrm{HI})$ for the DLAs, where the band centers and FWHM of each DIB are taken from Jenniskens \& Desert (1994). These models are not computed for the $\lambda 4428$ and $\lambda 6613$ DIBs since these DIBs have no published $N(\mathrm{HI})$ relationships. In the following sections we will discuss limits on reddening and gas-to-dust ratios. Thus, we also illustrate (thick curves) the expected observed DIB absorption profiles assuming an $E(B-V)=0.04$, the upper limit for high redshift DLAs assuming SMC-like extinction (Ellison et al. 2005). For the AO 0235+164 DLA, we adopted the measured $E(B-V)=0.23$ (Junkkarinen et al. 2004)). The computations of the reddening models are discussed in $\S[5.2$,

The equivalent widths of the DIBs estimated from the Galactic DIB- $N(\mathrm{HI})$ and Galactic DIB- $E(B-V)$ models (see Welty et al. 2006) are presented in Table 4. Columns 1-2 list the DLA number and the QSO with associated DLA redshift. Columns 4-8 provide the observed equivalent widths and equivalent width limits for the $\lambda 4428, \lambda 5780, \lambda 5797, \lambda 6284$, and $\lambda 6613$ DIBs. Also listed are the predicted equivalent widths of the DIBs, where $\mathrm{EW}[N(\mathrm{HI})]$ denotes the Galactic $N(\mathrm{HI})$ scaling and $\operatorname{EW}[E(B-V)]$ denotes the reddening scaling in $\mathrm{m} \AA$. $E(B-V)_{\text {lim }}$ is the reddening

\footnotetext{
${ }^{3}$ The VLT/UVES spectrum is not shown because only the $\lambda 4428$ DIB is covered. The UVES spectrum has a high resolution that makes identifying the broad $\lambda 4428$ DIB very difficult.
} 
upper limit for each DLA based upon the observed DIB equivalent width limits and the reddening relation (see $\S 5.2$ ).

\section{Results and Discussion}

In this section, we examine the DIB strengths in DLAs compared to the Galactic DIB- $N(\mathrm{HI})$ relation and the Galactic $E(B-V)$ relation. The former provides information on the gas content and the latter provides information on the reddening (an indirect indicator of dust content). Our observations allow us to estimate lower limits on the gas-to-dust ratios, $N(\mathrm{HI}) / E(B-V)$, of the DLAs in our sample.

\subsection{Gas Content}

The widely observed Galactic DIB- $N(\mathrm{HI})$ relation describes the correlation of the equivalent width of the $\lambda 5780, \lambda 5797$, and $\lambda 6284$ DIBs with the column density of neutral hydrogen along the same line of sight (Herbig 1995; Welty et al. 2006). Welty et al. (2006) extend the Galactic work by including Magellanic Cloud sightlines.

Plotted in Fig. 9 are the DIB-N(HI) relations for the $\lambda 5780$ (panel $a$ ), $\lambda 5797$ (panel $b$ ), and $\lambda 6284$ DIBs (panel $c$ ), where $\log N(\mathrm{HI})\left[\mathrm{cm}^{-2}\right]$ is plotted against the logarithm of the DIB equivalent widths $[\mathrm{m} \AA]$. Also plotted are the $(1 \sigma)$ error bars for measured values or downward arrows representing upper limits. The vertical error bars for the $\lambda 5780$ DIB detection in the AO $0235+164$ DLA are smaller than the point size. The solid lines represent the weighted best-fits to the Galactic data from Welty et al. (2006). The equivalent widths predicted at a given $N(\mathrm{HI})$, the $\operatorname{EW}[N(\mathrm{HI})]$ listed in Table 4 , are computed using this best-fit line and the $N(\mathrm{HI})$ of each DLA (Figs. 28 contain these models superimposed on the data). The dotted lines in Fig. 9 roughly enclose the regions containing the Galactic data. The dashed lines roughly enclose the regions containing the LMC data, and the dot-dashed lines roughly enclose the regions containing the SMC data.

The DIBs in the DLAs whose equivalent width limits in Fig. 9 lie below the Galactic best-fit lines are robust enough that we should have detected them if they followed the same dependence on $N(\mathrm{HI})$ as Galactic sightlines. In several DLAs, the limits are inconsistent with the expectations from the Galactic sightlines; the DIB strengths are weaker than expected. The DIB limits are consistent with the strengths of DIBs in the LMC or SMC. However, higher signal-to-noise data are required to determine whether the DIB strengths are actually consistent with or are even weaker than those observed in the LMC and SMC. The $\lambda 6284$ DIB, panel (c) of Fig. 9, provides the most stringent evidence that the DIB strengths in four DLAs are not consistent with DIB strengths in the Milky Way. The four DLAs toward AO 0235+164 $(z=0.524)$, Q0738+313 $(z=0.091)$, PKS 0952+179 $(z=0.239)$, and PKS 1127-145 $(z=0.313)$ are underabundant in their $\lambda 6284$ DIB 
strengths by factors of 4-10 times expected from the Galactic DIB- $N(\mathrm{HI})$ relation.

A number of environmental factors such as reddening, metallicity, and ionizing radiation may be responsible for DLAs not following the Galactic DIB- $N(\mathrm{HI})$ relation. Whether it is one or a combination of these factors, the data suggest that the environments probed by DLA sightlines differ from those within the Milky Way in which DIBs are present. The environments that give rise to the DIBs are likely very localized. Galactic properties vary even on small scales, which is why Cox et al. (2007) find varying differences and DIB strengths along different sightlines within the larger confines of the SMC. QSO sightlines probe relatively small transverse spatial scales in DLA galaxies. Therefore, our results do not eliminate the possibility that DIBs follow the Galactic DIB$N(\mathrm{HI})$ relationships elsewhere in these galaxies. We are less inclined to suggest that our results indicate redshift evolution of organics, since infrared emission from PAHs has been observed in the extremely dusty environments of ULIRGs as high as redshift $z \sim 2$ (Yan et al. 2005).

\subsection{Reddening}

There has been a long history of investigating DIB dependence on color excess (Merrill \& Wilson 1938; Herbig 1993). Whereas Galactic sight-lines along the disk (low Galactic latitudes) have typical reddening values of 0.1 to 1.0, DLAs typically have lower reddening. Murphy \& Liske (2004) use Sloan Digital Sky Survey (SDSS) QSO spectra to estimate $E(B-V)<0.02$ mag $(3 \sigma)$ along DLA sightlines at $z \sim 3$, assuming SMC-like extinction. Ellison et al. (2005) use radio selected QSOs from the Complete Optical and Radio Absorption Line System (CORALS) survey to estimate reddening along DLA sightlines. The CORALS survey avoids the potential problem of bias from optical luminosity selected samples that may inhibit the detection of more reddened QSOs. They find $E(B-V)<0.04(3 \sigma)$ assuming SMC-like extinction for $1.9 \leq z \leq 3.5$ DLAs. Using a sample of $0.8 \leq z \leq 1.3$ DLAs selected by CaII absorption, Wild \& Hewett (2005) estimate an average reddening of $E(B-V)=0.06$ assuming LMC and SMC extinction curves.

The correlations between reddening and the $\lambda 5780, \lambda 5797$, and $\lambda 6284$ DIB strengths are tight among Galactic and Magellanic Cloud sightlines, with best fits (Welty et al. 2006),

$$
\begin{aligned}
\log \mathrm{EW}[E(B-V)]_{\lambda 5780} & =0.99 \log E(B-V)+2.65, \\
\log \mathrm{EW}[E(B-V)]_{\lambda 5797} & =0.99 \log E(B-V)+2.26,
\end{aligned}
$$

and

$$
\log \mathrm{EW}[E(B-V)]_{\lambda 6284}=0.80 \log E(B-V)+3.08 .
$$

The rms scatter of the measured equivalent widths about these relationships are less than 0.15 dex; in other words, the $\lambda 5780, \lambda 5797$, and $\lambda 6284$ DIBs fairly equally obey the DIB $-E(B-V)$ relation in the Galaxy. Combining Galactic and extragalactic data, Ellison et al. (2008) find a $\lambda 5780$ DIB$E(B-V)$ relation with a slightly larger slope, 1.27, and intercept, 2.77. Compared to Welty et al. 
(2006), the Ellison et al. (2008) results yield a $10 \%$ difference in $\log \mathrm{EW}[E(B-V)]_{\lambda 5780}$ for unit reddening.

Correlations with the $\lambda 4428$ DIB equivalent width are notoriously problematic to compute because of the difficulty in measuring the DIB's broad width. Previous work has noted a correlation of the $\lambda 6613$ DIB equivalent width with $E(B-V)$ (Megier et al. 2005; Thorburn et al. 2003; Weselak et al. 2001). In the case of both the $\lambda 4428$ and $\lambda 6613$ DIBs, we employ the reddening relations provided by T.P. Snow (unpublished, private communication). Using the best-fit for 75 Galactic sightlines for $\lambda 4428$ and 123 Galactic sightlines for $\lambda 6613$,

$$
\mathrm{EW}[E(B-V)]_{\lambda 4428}=2093.99 E(B-V)
$$

and

$$
\operatorname{EW}[E(B-V)]_{\lambda 6613}=217.06 E(B-V) .
$$

The scatter about these relations is relatively large when compared to the other DIBs studied in this work. The $\lambda 4428$ DIB- $E(B-V)$ relation has a Pearson correlation coefficient of 0.825 , while the $\lambda 6613$ DIB- $E(B-V)$ relation has a Pearson correlation coefficient of 0.831 (unpublished, T.P. Snow, private communication).

Assuming the upper limit measured for high redshift DLAs of $E(B-V)=0.04$ from Ellison et al. (2005), we computed the expected DIB equivalent widths using Eqs. 1, 2, 3, 4, and 5. The only exception is the AO $0235+164$ DLA, which has a measured reddening of $E(B-V)=0.23$ (Junkkarinen et al. 2004). The expected DIB equivalent widths are listed in Table 4. Assuming these reddening values, the expected DIB equivalent widths are systematically smaller than those predicted by the DIB- $N$ (HI) relationship. In Figs. 28 we show the expected DIB profiles (thick curves). With the exception of the $\lambda 6284$ DIB limit measured from the spectrum of the DLA toward AO $0235+164$ and the $\lambda 4428$ DIB limit measured from the spectrum of the DLA toward PKS 1127-145, the expected DIB equivalent widths for adopted reddening values are smaller than our measured equivalent width limits; thus, if these reddening values are appropriate for intermediate redshift DLAs, we would not have detected these DIBs. As discussed in $\S$, the $\lambda 6284$ DIB limit for the AO $0235+164$ system is problematic due to a large atmospheric feature and the $\lambda 4428$ DIB limit for the PKS 1127-145 system is unreliable due to difficulties in continuum fitting the broad DIB within the high resolution VLT/UVES data.

Assuming the Galactic DIB- $E(B-V)$ relations are valid for DIBs in DLAs, we used our measured equivalent width limits for the $\lambda 4428, \lambda 5780, \lambda 5797, \lambda 6284$, and $\lambda 6613$ DIBs to compute upper limits on the reddening for the DLAs in our sample directly from the weighted slopes of the DIB- $E(B-V)$ relationships (Eqs. 1, 2, 3, 4, and 5) from Welty et al. (2006) and T.P. Snow (unpublished, private communication). The reddening limits, $E(B-V)_{\text {lim }}$, are listed in Table 4 for each DIB.

In Fig. 10, we illustrate the sensitivity of this method for $E(B-V)$ values of $0.02,0.04,0.10$, $0.20,0.40$, and 1.0, respectively. Plotted are $\log (\mathrm{EW} \mathrm{Limit} / \mathrm{EW}[E(B-V)])$ for each DLA for the 
$\lambda 4428, \lambda 5780, \lambda 5797, \lambda 6284$, and $\lambda 6613$ DIBs (panels $a, b, c, d, e$, respectively). For the condition $\log (\mathrm{EW}$ Limit/EW $[E(B-V)]) \leq 0$, the reddening predicted equivalent width for a given DIB is greater than or equal to the upper limits afforded by our data. A value of $\log (\mathrm{EW}$ Limit/EW $[E(B-$ $V)])=0$ corresponds to our computed reddening upper limit, $E(B-V)_{\lim }$, at which the DIB should just become detectable in our data. The vertical error bars define the $1 \sigma$ uncertainties in $\log (\mathrm{EW}$ Limit/EW $[E(B-V)])$ evaluated at our computed $E(B-V)_{\text {lim }}$. The uncertainties are calculated using standard error propagation taking into account the uncertainties in both the W Limit and the slope of the $\mathrm{EW}[E(B-V)]$ relations from Welty et al. (2006) and T.P. Snow (unpublished, private communication). The $\lambda 4428$ and $\lambda 5780$ DIBs for the DLA toward AO $0235+164$ are left blank because they are confirmed detections (Junkkarinen et al. 2004; York et al. 2006) with a known reddening of $E(B-V)=0.23$ (Junkkarinen et al. 2004).

Note that in Fig. 10, the $\lambda 6613$ DIB- $E(B-V)$ relation is the least constraining of the DIBs in determining the upper reddening limit, $E(B-V)_{\text {lim. }}$. Furthermore, due to the scatter in the $\lambda 4428$ and $\lambda 6613$ DIB- $E(B-V)$ relations, the errors in the best-fit slope in Eqs. 4 and 5 are relatively large which results in the large errors displayed in Fig. 10a and 10k. For these reasons, we do not adopt upper reddening limits, $E(B-V)_{\text {lim }}$, from the $\lambda 4428$ or $\lambda 6613$ DIBs.

Our final adopted reddening limit for a given DLA is determined from either the $\lambda 5780$ or $\lambda 6284$ DIBs. The adopted limits are listed in Table 5, Two of the DLAs have limits of $E(B-V) \leq$ 0.05 (Q0738+313 $z=0.091$ and PKS 0952+179), both from the $\lambda 6284$ DIB. The DLA toward B2 $0827+243$ has an upper limit to the reddening of $E(B-V) \leq 0.07$ from the $\lambda 5780$ DIB (the $\lambda 6284 \mathrm{DIB}$ is not covered). The $\lambda 6284 \mathrm{DIB}$ provides upper reddening limits for the remaining DLAs toward Q1229-020 $(E(B-V) \leq 0.08)$, Q0738+313 $z=0.221(E(B-V) \leq 0.14)$, and PKS 1127-145 $(E(B-V) \leq 0.21)$. If we were to apply constraints from the $\lambda 4428 \mathrm{DIB}$, we would infer upper reddening limits of 0.07, 0.04, and 0.03 for the $z=0.221$ DLA toward Q0738+313, and the DLAs toward B2 $0827+243$ and PKS 1127-145, respectively.

For the DLA at $z=0.524$ toward AO $0235+164$, there are two detections from York et al. (2006) (the $\lambda 5705$ and $\lambda 5780$ DIBs) and one detection from Junkkarinen et al. (2004) (the $\lambda 4428$ DIB). The equivalent widths measured from the $\lambda 4428$ and $\lambda 5780$ DIBs are larger than expected from the measured $E(B-V)=0.23$ (Junkkarinen et al. 2004). The $\lambda 5797$ and $\lambda 6613$ DIBs give limits consistent with this, but the $\lambda 6284$ DIB suggests an $E(B-V) \leq 0.06$ by this technique. Essentially, we should have discovered the $\lambda 6284$ DIB assuming our technique is correct because Junkkarinen et al. (2004) measures an $E(B-V)=0.23 \pm 0.01$.

A plausible reason for our non-detection of the $\lambda 6284$ DIB in the AO $0235+164$ DLA is that the expected position of the $\lambda 6284$ DIB at $z=0.524$ resides directly in a broad atmospheric absorption band (see panel $b$ in Fig. 2). York et al. (2006) attempted to overcome this by modeling the $\lambda 6284$ DIB with various equivalent widths and convolving it with the atmospheric band. The limit they achieve (see Table 3) is based on the minimum equivalent width needed to separate the DIB out of the atmospheric band. This is a robust way to measure the limit. However, there is some inherent 
difficulty in this method because it has to be assumed a priori that the atmospheric band is not contaminated by DIB absorption. A second potential reason that we do not detect the $\lambda 6284$ DIB in the $\mathrm{AO} 0235+164$ DLA, given its high reddening, is that this DIB may not follow the Galactic DIB- $E(B-V)$ relation in DLAs. Indeed, in the Magellanic Clouds, $\lambda 6284$ DIBs are weaker than their Galactic counterparts by a factor of two relative to the Galactic $\lambda 6284$ DIB- $E(B-V)$ relation (Welty et al. 2006). Given that the $\lambda 6284$ DIBs in four of our DLAs are constrained to be $4-10$ times weaker than the Galactic $\lambda 6284$ DIB- $N$ (HI) relation, it is reasonable that DIB relations in DLAs could be more Magellanic Cloud-like than Galactic-like.

Our observations have provided reddening constraints of $0.05 \leq E(B-V) \leq 0.08$ for four DLAs at $0.09 \leq z \leq 0.52$. This extends to lower redshift the work of Ellison et al. (2005), who report $E(B-V) \leq 0.04$ for $1.9 \leq z \leq 3.5$ DLAs, and of Murphy \& Liske (2004), who report $E(B-V) \leq 0.02$ for $z \sim 3$ DLAs (all limits are $3 \sigma$ ). Reddening is an indirect measure of dust content. The slope of any DIB- $E(B-V)$ relation likely has a strong dependence on the nature of the dust (particle size, abundance, and composition). Junkkarinen et al. (2004) argue that the dust in the AO 0235+164 DLA is more Galactic-like than Magellanic-like, but with fewer small particles. Because the organics responsible for the DIBs may depend on dust for formation and/or survival, the abundance and nature of the dust in the DLA toward AO 0235+164 might be responsible for the presence of DIBs observed in this DLA.

\subsection{Gas-to-Dust Ratios}

Assuming that our method of estimating $E(B-V)_{\text {lim }}$ realistically reflects the upper limit on reddening in our DLAs, we can estimate lower limits in their gas-to-dust ratios, $N(\mathrm{HI}) / E(B-V)$. We present the gas-to-dust ratio lower limits in Table 5 they are computed using the measured $N(\mathrm{HI})$ from the literature and our adopted upper $E(B-V)$ limits. The lower limits range from $2.9 \times 10^{21}$ to $42 \times 10^{21} \mathrm{~cm}^{-2} \mathrm{mag}^{-1}$. Note that the gas-to-dust ratio $19.2 \times 10^{21} \mathrm{~cm}^{-2} \mathrm{mag}^{-1}$ for the $z=0.524$ DLA toward AO 0235+164 was measured by Junkkarinen et al. (2004).

Bouchet et al. (1985) measure lower and upper values, $37 \times 10^{21}$ and $52 \times 10^{21} \mathrm{~cm}^{-2} \mathrm{mag}^{-1}$, respectively, for SMC gas-to-dust ratios. Gordon et al. (2003) determined a gas-to-dust ratio of $19.2 \times 10^{21} \mathrm{~cm}^{-2} \mathrm{mag}^{-1}$ for the LMC (from their LMC-2 data). The average LMC sample of Gordon et al. (2003) yields $11.1 \times 10^{21} \mathrm{~cm}^{-2} \mathrm{mag}^{-1}$. A linear fit by Cox et al. (2006b) to their LMC data gives a similar gas-to-dust ratio of $14.3 \times 10^{21} \mathrm{~cm}^{-2} \mathrm{mag}^{-1}$. For the Milky Way, Bohlin et al. (1978) find a gas-to-dust ratio of $4.8 \times 10^{21} \mathrm{~cm}^{-2} \mathrm{mag}^{-1}$; whereas, Cox et al. (2006b) find a ratio of $4.03 \times 10^{21} \mathrm{~cm}^{-2} \mathrm{mag}^{-1}$ from the fit to their Galactic data.

Although the SMC, LMC, and Milky Way appear to have distinct ranges of gas-to-dust ratios, in reality, there is a continuum that likely reflects the variation of local environments in each. Gordon et al. (2003) measure $E(B-V)$ and $N(\mathrm{HI})$ toward four stars in the bar of the SMC and one star in the wing of the SMC. From these data, the gas-to-dust ratios in the SMC bar span 
the range $17-51 \times 10^{21} \mathrm{~cm}^{-2} \mathrm{mag}^{-1}$. These values overlap with both the LMC gas-to-dust ratios of Gordon et al. (2003) and Cox et al. (2006b) and the SMC gas-to-dust ratios of Bouchet et al. (1985). The SMC wing data yield $15.2 \times 10^{21} \mathrm{~cm}^{-2} \mathrm{mag}^{-1}$, which falls very near the Cox et al. (2006b) LMC fit.

Cox et al. (2007) searched for DIBs toward six lines of sight in the bar and wing of the SMC. The sightline AzV456, which is in the wing of the SMC, is the only sightline in which they detected DIBs. From their $E(B-V)$ and $N(\mathrm{HI})$ data (Table 9 on-line material), the gas-to-dust ratio in the SMC wing is $\sim 7$ times lower $\left(\sim 7.4 \times 10^{21} \mathrm{~cm}^{-2} \mathrm{mag}^{-1}\right)$ than the gas-to-dust ratio in the SMC bar $\left(\sim 52.4 \times 10^{21} \mathrm{~cm}^{-2} \mathrm{mag}^{-1}\right)$. This SMC wing value lies between the Bohlin et al. (1978) and Cox et al. (2006b) Milky Way and the Gordon et al. (2003) and Cox et al. (2006b) LMC gasto-dust ratios. The SMC bar value lies just above the upper range of the Bouchet et al. (1985) SMC gas-to-dust ratio. It is of interest to note that Cox et al. (2007) detected DIBs only in the sightline with the smallest gas-to-dust ratio in their sample and that this ratio approaches the range observed for the Milky Way.

Cox et al. (2007) argue that the SMC wing is quiescent while the star formation regions of the SMC bar are turbulent and exposed to larger UV fluxes. Welty et al. (2006) calculate an increase of $\sim 28-83$ times the average interstellar radiation field (ISRF) for sightlines in the SMC bar near star-forming HII regions; whereas, the SMC wing is more similar to the Galaxy with a radiation level of $\sim 0.6$ times the average ISRF. The variation in the gas-to-dust ratios within the SMC are likely a reflection of the balance of dust formation processes, such as accretion, with dust destruction (Cox et al. 2007). Thus, the dust, and possibly the organics, may be destroyed in the SMC bar explaining why Cox et al. (2007) could not detect DIBs in their sample of SMC bar stars.

In Fig. 11 we plot $\log N(\mathrm{HI})$ versus $\log E(B-V)_{\lim }$ for the DLAs in our sample. The $N(\mathrm{HI})$ data points are taken from Table 1 and the $E(B-V)_{\lim }$ are taken from Table 5, The $\log N(\mathrm{Hi})$ error bars are $1 \sigma$. The leftward arrows represent reddening upper limits for our sample. For Q0738+313 at $z=0.221$, B2 $0827+243$, and PKS 1127-145, the $\lambda 4428$ DIB provides more stringent limits on $E(B-V)_{\text {lim. }}$. However, they should be considered less robust due to the large errors as shown in Fig. 10, panels $a$ and $e$.

Although the gas-to-dust ratios in the SMC, LMC, and Milky Way exhibit some overlap, in general the Milky Way ISM values tend to be lower than those of the LMC which tend to be lower than those of the SMC (Bohlin et al. 1978; Bouchet et al. 1985; Gordon et al. 2003; Cox et al. 2006b). In Fig. 11, the dot-dashed lines illustrate the upper and lower SMC values of Bouchet et al. (1985). The middle three lines in Fig. 11 represent gas-to-dust ratios for the LMC, and the bottom two lines represent those for the Galaxy. The long-dashed line gives the LMC-2 gas-to-dust ratio derived from Gordon et al. (2003). The Cox et al. (2006b) ratio for the LMC is given by the dot-dot line. The short-dashed line is the Gordon et al. (2003) ratio from their average LMC sample. The dashed MW line is the Bohlin et al. (1978) ratio, and the dotted MW line is the Cox et al. (2006b) ratio. 
As shown in Fig. 11, the DLA toward PKS 0952+179 has a gas-to-dust ratio that is consistent with or greater than the SMC. Two of the DLAs in our sample, Q0738+313 ( $z=0.091)$ and PKS 1127-145, have ratios consistent with or greater than the LMC gas-to-dust ratios. Two, Q0738+313 $(z=0.221)$ and Q1229-020, are consistent with or greater than the Galactic ratios. The ratio for $\mathrm{B} 20827+243$, which has the lowest $N(\mathrm{HI})$ in our sample, is not well constrained. The measured ratio for the $z=0.524$ DLA toward AO $0235+164$ is $N(\mathrm{HI}) / E(B-V)=19.2 \times 10^{21}$ $\mathrm{cm}^{-2} \mathrm{mag}^{-1}$ (Junkkarinen et al. 2004), which is consistent with the LMC-2 ratio of Gordon et al. (2003) and four times larger than the Galactic ratio from Bohlin et al. (1978). This DLA has a very large $N(\mathrm{HI})$, and the largest $E(B-V)$ (Junkkarinen et al. 2004) of the DLAs presented here.

Three DLAs are constrained to have slightly larger gas-to-dust ratios than the $z=0.524$ DLA toward AO $0235+164$, the $z=0.091$ DLA toward Q0738+313 ( $\simeq 1.5$ times greater), $z=0.239$ DLA toward PKS 0952+179 ( $\simeq 2.2$ times greater), and the $z=0.313$ DLA toward PKS 1127-145 $\left(\simeq 1.2\right.$ times greater). However, for the DLA toward PKS 1127-145, if we apply $E(B-V)_{\lim }$ from the $\lambda 4428 \mathrm{DIB}$, the gas-to-dust ratio is $\simeq 7$ times greater than that of the AO $0235+164$ DLA and would be more consistent with gas-to-dust ratios greater than measured in the SMC. Again, we caution that this more stringent ratio is not adopted due to large errors from the scatter in the $\lambda 4428$ DIB- $E(B-V)$ relation (see Eq. 4).

The sightlines toward DLAs are certainly gas rich, which they share with Galactic sightlines that contain DIBs. However, their environments, as probed by the background QSO, may have large variations in $E(B-V)$, metallicity, and radiation. Understanding the magnitudes and properties of the dust, radiation, $\mathrm{HI}$ and $\mathrm{H}_{2}$ content, and metallicity in DLA samples holds promise of revealing whether the environments of DLAs are conducive for the organics that give rise to DIBs. Reddening is seemingly low in the sightlines probing the galaxies in this sample, which may play a pivotal role in inhibiting DIB strengths. The strengths of DIBs in DLAs do not follow the Galactic $N(\mathrm{HI})$ relation (see Fig. 9). However, the $\lambda 5780$ detection in the AO $0235+164$ DLA is consistent with expectations from the DIB- $E(B-V)$ relation for Galactic and extragalactic points (Ellison et al. 2008). Thus, gas-to-dust ratio may not be as good a predictor as reddening for the presence of DIBs in DLAs. This would suggest that DIBs might be present in DLAs with a wide range of gas-to-dust ratios, but that they lie within the right-hand region of the $\log N(\mathrm{HI})-\log E(B-V)$ plane of Fig. 11.

Ellison et al. (2005) conclude that their sample of $1.9 \leq z \leq 3.5$ high redshift DLAs does not follow a Galactic gas-to-dust relation, which is consistent with what we find for four of the DLAs in this work ( $\mathrm{AO} 0235+164$ at $z=0.524$, Q0738+313 at $z=0.091$, PKS 0952+179 at $z=0.239$, and PKS 1127-145 at $z=0.313)$. However, as found by Ellison et al. (2005), we cannot conclude whether the gas-to-dust ratios in our lower redshift DLAs are consistent with the SMC gas-to-dust ratios. The remaining three DLAs (Q0738+313 at $z=0.221$, B2 0827+243, and Q1229-020) do not have sufficient reddening limits to compare with the high redshift sample of Ellison et al. (2005). 


\subsection{Metallicity versus Reddening}

Cox et al. (2007) give evidence that metallicity plays an important role in DIB strength since a high carbon abundance is necessary for the creation of the organic molecules and the dust grains on which they may be formed. In Table 1, we list the zinc and iron abundances from the literature for our DLA sample. Zinc is a good metallicity indicator because it traces iron-group abundances and does not readily deplete on dust. Unfortunately, only upper limits on zinc abundances have been measured, with the exception of the Q1229-020 DLA, where $[\mathrm{Zn} / \mathrm{H}]=-0.47$ (Boissé et al. 1998). Thus, we cannot address any trends of DIB strengths with metallicity.

The Q1229-020 DLA has a metallicity roughly 0.5 dex above the average metallicity of DLAs, and its metallicity is consistent with that of the AO $0235+164$ DLA. Since it has a relatively stringent upper limit on the reddening, $E(B-V)_{\text {lim }} \leq 0.08$, much below the $E(B-V)=0.23$ of the AO $0235+164$ DLA, it may be a promising candidate for contributing to an understanding of the role of metallicity in determining DIB strengths in DLAs. If metallicity plays a role, it is expected that DIB strengths in DLAs would to some degree scale with $N(\mathrm{HI})$. The $N(\mathrm{HI})$ of the Q1229-020 DLA is a full dex below that of the AO 0235+164 DLA. Thus, that our upper limit on the equivalent width of the $\lambda 5780$ DIB in the Q1229-020 DLA is a factor of 1.7 below that detected for the AO $0235+164$ DLA is not constraining. A very deep spectrum of the DIBs in the Q1229-020 DLA would be very interesting.

Selecting DLAs by metal absorption would represent another approach to searching for DIBs in external galaxies, since these DLAs may represent a more reddened population. Wild et al. (2006) propose that CaII absorbers may represent an intermediate link between the quiescent, metal-poor and dust-poor DLAs and the intermediate redshift, star-forming, and metal-rich Lyman break galaxies with typical reddening of $E(B-V)=0.15$ to 0.20 . Ellison et al. (2008) searched for DIBs in nine $0.07 \leq z \leq 0.55$ CaII-selected absorbers, and detected the $\lambda 5780$ DIB in only one. At this early juncture, the CaII selection success rate is comparable to that of HI-selected DLAs. MgII absorbing DLAs may also represent a slightly more reddened population of DLAs (Ménard et al. 2008).

\section{Conclusions}

In this paper we employ a generalized method of the Schneider et al. (1993) technique to find lines and determine conservative equivalent width limits for DIBs in seven DLAs along with an assessment of uncertainties in these limits. We find:

(1) The $\lambda 6284$ DIB in four of the DLAs in our sample have equivalent width upper limits that are 4-10 times lower then expected for similar $N(\mathrm{HI})$ relative to Galactic sight lines. These limits are not inconsistent with the $\lambda 6284$ DIB strengths found in the LMC and SMC.

(2) Assuming the $\lambda 5780$ and $\lambda 6284$ DIB- $E(B-V)$ relations hold for DLAs, as it does for 
Galactic and Magellanic Cloud sightlines, we estimated upper limits on the reddening for our sample. In four of our DLAs we estimate $E(B-V) \leq 0.08$ with two DLAs having an $E(B-V) \leq$ 0.05. These results are consistent with high redshift DLA samples, which have $E(B-V)<0.04$ (Ellison et al. 2005) and $E(B-V)<0.02$ (Murphy \& Liske 2004).

(3) Applying the $E(B-V)$ limits, one of our DLAs is consistent with having the same or larger gas-to-dust ratio as the SMC. Two of our DLAs are consistent with having gas-to-dust ratios at least as large as sightlines in the LMC. Three of our DLAs have less stringent limits that give lower limit gas-to-dust ratios consistent with Galactic, LMC, or SMC sightlines. The AO 0235+164 DLA has a measured $E(B-V)$ and $N(\mathrm{HI})$ that puts its gas-to-dust fraction on the high end of the LMC sightlines as stated in other work (Junkkarinen et al. 2004). The $E(B-V)_{\text {lim }} \leq 0.06$ constrained from the $\lambda 6284$ DIB is inconsistent with the known $E(B-V)=0.23$ measured in Junkkarinen et al. (2004). We should have been able to detect the $\lambda 6284$ DIB in the AO $0235+164$ DLA given the limit adopted by York et al. (2006). Three possibilities are that our method of determining the $E(B-V)_{\lim }$ does not apply to DLAs, our limits do not adequately take into account the large atmospheric absorption band (see Fig. 2b), or that conditions are not favorable to the formation or survival of this DIB carrier.

It is interesting to speculate whether ionization conditions may be an important factor in inhibiting or enhancing DIB strengths. Welty et al. (2006) test the ionization effects of DIBs along Galactic, LMC, and SMC lines of sight. However, they do not find any significant trends. On the contrary, Cox et al. (2007) measure the UV radiation field along lines of sight toward the SMC and find UV radiation is an important environmental factor in DIB strengths. Laboratory spectroscopists claim that the DIBs may be due to partially ionized PAHs because they produce a wealth of absorption features in the optical spectrum (Snow 2001). If this is the case then a significant UV radiation may be required. However, UV radiation that is too high will dissociate the molecules. In this work, we are unable to explore the affects of ionization conditions; knowledge of ionization conditions in specific DLAs is difficult to obtain.

It is also interesting to speculate whether metallicity may be an important factor in inhibiting or enhancing DIB strengths. However, since we have robust metallicity measurements for only two of the DLAs in our sample, we cannot directly address the affects of metallicity. We do point out that obtaining deep spectra of DIBs in DLAs with known metallicity could be a fruitful future research direction, especially if reddening were also known. A direct comparison between the low metallicity Q1229-020 DLA and the high metallicity AO 0235+164 DLA might be fruitful as a first examination of the affects of metallicity in determining what governs DIB strengths in DLAs.

\subsection{Implications of our Results}

Our results imply that reddening is a more crucial indicator of DIB strengths than is H I content for DLAs; the low reddening in DLA selected galaxies inhibits the presence of DIBs. However, our 
sample is small and additional observations are required to ascertain the strength of this statement (metallicity and ionization conditions likely play a role as well).

The weakness of DIB strengths in our sample hints that the environments of high $N(\mathrm{HI})$ DLAselected galaxies may be less suitable to create and/or sustain the organic molecules than those of the Galaxy. Not only is the immediate solar environment beneficial for sustaining life, but it may be that the Galaxy is a more hospitable location for the survival of organic molecules that may have been the precursors to biology on Earth. If these molecules are important as precursors to life in the universe, charting their presence to high redshift places constraints on how long ago and in which environments life could have potentially formed. The presence of DIBs in the AO 0235+164 DLA demonstrates that organic molecules existed in at least one DLA selected environment at a redshift of $z \sim 0.5$, or some 5 Gyrs ago (York et al. 2006).

Due to their weakness relative to DIBs in the Galaxy, observing DIBs in the general population of high redshift galaxies (as opposed to ULIRGs and star bursting galaxies) remains a challenge. Selecting galaxies by DLA absorption may yet prove to be a lucrative method for detecting DIBs at high redshifts, provided the detection sensitivity can be increased. However, most known DLAs reside at high redshift where the DIBs move into the near-IR, where high sensitivity spectroscopy is time intensive.

BL acknowledges support via NASA's Graduate Student Researchers Program (GSRP grant NNG04GN55H). CWC acknowledges NSF grant AST-0708210. BAY acknowledges the support of NSERC via their post-graduate scholarship (PGS) program, and TPS acknowledges NASA grant NNG04GL34G for partial support of this work. Some data were obtained from the Gemini telescopt 4 under program GS-2004A-Q-32. We thank M. Murphy (Swinburne University of Technology) for the VLT/UVES spectrum and W.L.W. Sargent (Caltech) for the Keck/HIRES spectrum used in this project. We also acknowledge Glenn Kapcrzak (NMSU) for contributed computations.

Facilities: VLT(FORS2, UVES), APO(DIS), Keck(HIRES), WHT(ISIS), Gemini(GMOS).

\footnotetext{
${ }^{4}$ Based on observations obtained at the Gemini Observatory, which is operated by the Association of Universities for Research in Astronomy, Inc., under a cooperative agreement with the NSF on behalf of the Gemini partnership: the National Science Foundation (United States), the Science and Technology Facilities Council (United Kingdom), the National Research Council (Canada), CONICYT (Chile), the Australian Research Council (Australia), CNPq (Brazil) and SECYT (Argentina)
} 


\section{A. Appendix}

\section{A.1. Measuring Equivalent Width Detection Thresholds}

A straight forward method for determining equivalent width limits was presented by Lanzetta et al. (1987). The uncertainty (or error) spectrum is used to compute the equivalent width limit in each individual pixel, $i$,

$$
\sigma_{w_{i}}=D_{i} \frac{E\left(\lambda_{i}\right)}{I_{c}\left(\lambda_{i}\right)}
$$

where $D_{i}$ is the pixel dispersion in angstroms, $E\left(\lambda_{i}\right)$ is the uncertainty in the flux at $\lambda_{i}$, and $I_{c}\left(\lambda_{i}\right)$ is the estimated continuum flux at $\lambda_{i}$. The $1 \sigma$ equivalent width detection threshold (limit) centered at $\lambda_{i}$ is then obtained by summing the individual equivalent width limits of adjacent pixels over a selected aperture,

$$
\sigma_{E W}\left(\lambda_{i}\right)=\left[\sum_{j=j_{1}}^{j_{2}} \sigma_{w_{j}}^{2}\right]^{1 / 2}
$$

where $j_{1}=i-m / 2, j_{2}=i+m / 2$, and where $m$, the aperture size, is an even number of pixels over which the absorption feature is anticipated. For unresolved features, $m$ can be taken to be roughly two resolution elements (i.e., roughly twice the number of pixels of the full-width half maximum of an unresolved line). Clearly, Eq. A2 can be generalized for resolved, broader features by increasing $m$ to span roughly twice the full-width half maximum of the anticipated broad line. The aperture summation method of Lanzetta et al. (1987) assigns equal weight to all pixels included in the summation around $\lambda_{i}$. This can result in an overestimate of the equivalent width detection threshold of the data.

Schneider et al. (1993) properly treat the relative weighting of pixels adjacent to $\lambda_{i}$ by weighting these pixels by the instrumental spread function, ISF. The ISF is then a probability weighting function of the flux centered at $\lambda_{i}$ with pixel weighting, $P_{j}$, defined such that

$$
\sum_{j=1}^{m} P_{j}=1
$$

where $m=2 j_{o}+1$, and where $j_{o}$ is a positive integer. For unresolved features, the ISF, and therefore the relative values of the $P_{j}$, can be taken as a Gaussian function characterized by the Gaussian width $\sigma_{\mathrm{ISF}}=\Delta \lambda_{i} / 2.35$, where $\Delta \lambda_{i}=\lambda_{i} / R$ is the full-width at half maximum of the ISF centered at $\lambda_{i}$ for a spectrograph with resolution $R$. The value of $j_{o}$ is chosen appropriately for the ISF such that $P_{1}=P_{m} \simeq 0$ (effectively making sure that the tails of the probability function are sampled out to where the probabilities vanish).

The $1 \sigma$ equivalent width detection threshold for unresolved features is then computed using

$$
\sigma_{E W}\left(\lambda_{i}\right)=D_{i}\left[\sum_{j=1}^{m} P_{j}^{2} E^{2}\left(\lambda_{k}\right) / I_{c}^{2}\left(\lambda_{k}\right)\right]^{1 / 2}\left[\sum_{j=1}^{m} P_{j}^{2}\right]^{-1},
$$


where $k=i+(j-1)-j_{o}$ is the "convolution index". The elements of the probability weighting function are given by

$$
P_{j}=\frac{\Phi_{j}}{\sum_{j=1}^{m} \Phi_{j}}
$$

where

$$
\Phi_{j}=\exp \left\{-\frac{\left(\lambda_{k}-\lambda_{i}\right)^{2}}{2 \sigma_{\mathrm{ISF}}^{2}}\right\} .
$$

If the feature is redshifted, then Eq. A4 must be divided by the factor $1+z$ to obtain the rest-frame equivalent width limit.

We have generalized Eq. A4 to (1) account for resolved features of known FWHM, and (2) account for regions in which problematic sky-line subtraction in the vicinity of $\lambda_{i}$ renders the data less certain than quantified by the uncertainty spectrum, $E(\lambda)$. We also explicitly include redshift dependence.

To account for resolved features, the $\sigma_{\text {ISF }}$ in Eq. A6 is replaced with the Gaussian width of the line spread function (LSF) of the redshifted resolved feature, which is

$$
\sigma_{\mathrm{LSF}}=\left[\left(\frac{\operatorname{FWHM}(1+z)}{2.35}\right)^{2}+\sigma_{\mathrm{ISF}}^{2}\right]^{1 / 2},
$$

where FWHM is the rest-frame full-width at half maximum of the feature, and $z$ is the redshift. We have assumed that both the intrinsic line shape and the ISF are well approximated by Gaussian functions.

To account for large residuals from problematic sky-line subtraction, the error in the flux, $E\left(\lambda_{k}\right)$, in Eq. A4 is replaced by $\hat{E}\left(\lambda_{k}\right)$, which is determined by the quality of the data at pixel $k$,

$$
\hat{E}\left(\lambda_{k}\right)=\left\{\begin{array}{lll}
\left|r\left(\lambda_{k}\right)\right| & \text { for } & \left|r\left(\lambda_{k}\right)\right| \geq 3 E\left(\lambda_{k}\right), \\
E\left(\lambda_{k}\right) & \text { for } & \left|r\left(\lambda_{k}\right)\right|<3 E\left(\lambda_{k}\right) .
\end{array}\right.
$$

where $\left|r\left(\lambda_{k}\right)\right|$ is the residual of the flux, $I\left(\lambda_{k}\right)$, with respect to the continuum,

$$
\left|r\left(\lambda_{k}\right)\right|=\left|I\left(\lambda_{k}\right)-I_{c}\left(\lambda_{k}\right)\right|
$$

Using the residual gives a more conservative estimate of the equivalent width detection threshold. The residual of a given pixel is used whenever the flux significantly deviates from the continuum. In addition to poor sky-line subtraction, sources of this deviation can be large telluric features, blending with absorption features, or a problematic continuum fit. Using the following substitution for the normalized flux error simplifies the equations for further analysis,

$$
Y\left(\lambda_{k}\right)=\frac{\hat{E}\left(\lambda_{k}\right)}{I_{c}\left(\lambda_{k}\right)}
$$


Applying these criteria transforms Eq. A4 from Schneider et al. (1993) into the rest-frame equivalent width detection threshold calculation used in this work,

$$
\sigma_{E W}\left(\lambda_{i}\right)=\frac{D_{i}}{(1+z)}\left[\sum_{j=1}^{m} P_{j}^{2} Y^{2}\left(\lambda_{k}\right)\right]^{1 / 2}\left[\sum_{j=1}^{m} P_{j}^{2}\right]^{-1}
$$

The rest-frame equivalent width limits presented in Table 3 are quoted at the $3 \sigma$ level.

\section{A.2. Uncertainty Assessment}

To quantify the quality of the equivalent width limits, we estimated the uncertainties in the $\sigma_{E W}\left(\lambda_{i}\right)$. The equivalent width limit in Eq. A11 explicitly includes the spectrograph resolution, $R$, the absorption line FWHM, the continuum fit, and the central wavelength of the absorption line, $\lambda_{l}$. Assuming that the uncertainties in these quantities can be estimated and are normally distributed, the variances in the equivalent width limits are obtained from

$$
V_{\sigma_{E W}}=\left[\frac{\partial \sigma_{E W}}{\partial R} \delta R\right]^{2}+\left[\frac{\partial \sigma_{E W}}{\partial \mathrm{FWHM}} \delta \mathrm{FWHM}\right]^{2}+\left[\frac{\partial \sigma_{E W}}{\partial I_{c}\left(\lambda_{k}\right)} \delta I_{c}\left(\lambda_{k}\right)\right]^{2}+\sigma_{\lambda_{l}}^{2}\left(\sigma_{E W}\right),
$$

where $\delta R$ is the uncertainty in the resolution, $\delta \mathrm{FWHM}$ is the uncertainty in the FWHM of the line, $\delta I_{c}$ is the uncertainty in the continuum, and $\sigma_{\lambda_{l}}\left(\sigma_{E W}\right)$ is the standard deviation in the equivalent width limit due to uncertainty in the wavelength center of the line. The terms in Eq. A12 for which explicit indices appear are computed at the center pixel, $i$, of the feature.

\section{A.2.1. Uncertainty in Resolution}

The uncertainty in the resolution, $\delta R$, can be obtained by measuring the full-width half maxi-

mum of unresolved sky lines and determining the standard deviation. Applying the chain rule, the partial derivative of the equivalent width limit with respect to resolution is

$$
\frac{\partial \sigma_{E W}}{\partial R}=\frac{\partial \sigma_{E W}}{\partial P_{j}} \frac{\partial P_{j}}{\partial \sigma_{\mathrm{LSF}}} \frac{\partial \sigma_{\mathrm{LSF}}}{\partial R},
$$

where,

$$
\begin{aligned}
\frac{\partial \sigma_{E W}}{\partial P_{j}} & =\frac{D_{i}}{(1+z)}\left[\frac{C^{1 / 2}}{G^{2}}\right]\left[\frac{1}{2} \frac{A G}{C}-B\right], \\
\frac{\partial P_{j}}{\partial \sigma_{\mathrm{LSF}}} & =P_{j}\left(Q_{j}-T / S\right), \\
\frac{\partial \sigma_{\mathrm{LSF}}}{\partial R} & =-\frac{1}{\sigma_{\mathrm{LSF}} R^{3}}\left(\frac{\lambda_{i}}{2.35}\right)^{2},
\end{aligned}
$$


and where,

$$
\begin{array}{rlrl}
A & =\sum_{j=1}^{m} 2 P_{j} Y^{2}\left(\lambda_{k}\right) & Q_{j} & =\frac{\left(\lambda_{k}-\lambda_{i}\right)^{2}}{\sigma_{\mathrm{LSF}}^{3}} \\
B & =\sum_{j=1}^{m} 2 P_{j} & T & =\sum_{j=1}^{m} \frac{\left(\lambda_{k}-\lambda_{i}\right)^{2}}{\sigma_{\mathrm{LSF}}^{3}} \exp \left\{-\frac{\left(\lambda_{k}-\lambda_{i}\right)^{2}}{2 \sigma_{\mathrm{LSF}}^{2}}\right\} \\
C & =\sum_{j=1}^{m} P_{j}^{2} Y^{2}\left(\lambda_{k}\right) & S & =\sum_{j=1}^{m} \exp \left\{-\frac{\left(\lambda_{k}-\lambda_{i}\right)^{2}}{2 \sigma_{\mathrm{LSF}}^{2}}\right\} \\
G & =\sum_{j=1}^{m} P_{j}^{2} .
\end{array}
$$

Again, the terms $P_{j}$ and $Q_{j}$ are evaluated at the center pixel, $i$, of the feature, $j=j_{o}$. Note that $Q_{j}$ vanishes at the line center.

\section{A.2.2. Uncertainty in FWHM}

In the case where the FWHM has a known uncertainty, $\delta \mathrm{FWHM}$, the affect on our equivalent width limits can be calculated. Again, applying the chain rule, the partial derivative of the equivalent width limit with respect to FWHM is

$$
\frac{\partial \sigma_{E W}}{\partial \mathrm{FWHM}}=\frac{\partial \sigma_{E W}}{\partial P_{j}} \frac{\partial P_{j}}{\partial \sigma_{\mathrm{LSF}}} \frac{\partial \sigma_{\mathrm{LSF}}}{\partial \mathrm{FWHM}}
$$

where $\partial \sigma_{E W} / \partial P_{j}$ and $\partial P_{j} / \partial \sigma_{\mathrm{LSF}}$ are given by Eqs. A14 and A15, From Eq. A7,

$$
\frac{\partial \sigma_{\mathrm{LSF}}}{\partial \mathrm{FWHM}}=\left(\frac{1+z}{2.35}\right)^{2} \frac{\mathrm{FWHM}}{\sigma_{\mathrm{LSF}}} .
$$

\section{A.2.3. Uncertainty in Continuum Placement}

We adopt the method of Sembach et al. (1991) to calculate the uncertainty in the continuum, $\delta I_{c}\left(\lambda_{k}\right)$, which is obtained directly from the data by taking the rms of the residuals about the continuum, $\sigma_{c}$, from $j=1$ to $m$ and multiplying by 0.5 . Numerical simulations suggest that this

provides a conservative estimate of the errors associated with the continuum (Sembach et al. 1991). The resulting uncertainty is

$$
\delta I_{c}\left(\lambda_{k}\right)=0.5 \sigma_{c}\left(\lambda_{k}\right),
$$

The partial derivative of the equivalent width limit with respect to the continuum is

$$
\frac{\partial \sigma_{E W}}{\partial I_{c}\left(\lambda_{k}\right)}=\frac{1}{2} \frac{D_{i}}{(1+z)} G^{-1} U^{-1 / 2} X
$$


where,

$$
\begin{aligned}
G & =\sum_{j=1}^{m} P_{j}^{2}, \\
U & =\sum_{j=1}^{m} P_{j}^{2} Y^{2}\left(\lambda_{k}\right), \\
X & =\sum_{j=1}^{m} \begin{cases}-2 P_{j}^{2} Y^{2}\left(\lambda_{k}\right) I_{c}^{-1}\left(\lambda_{k}\right) & \text { for } \hat{E}\left(\lambda_{k}\right)=E\left(\lambda_{k}\right), \\
\left|2 P_{j}^{2} I\left(\lambda_{k}\right) I_{c}^{-2}\left(\lambda_{k}\right)\left(1-I\left(\lambda_{k}\right) I_{c}^{-1}\left(\lambda_{k}\right)\right)\right| & \text { for } \hat{E}\left(\lambda_{k}\right)=\left|r\left(\lambda_{k}\right)\right| .\end{cases}
\end{aligned}
$$

The $j$ elements that are selected for the computation of $X$ depend on whether one uses the flux error, $E\left(\lambda_{k}\right)$, or the residual, $r\left(\lambda_{k}\right)$, (see Eq. A9) for a given pixel. The continuum contributes a large fraction of the error in the equivalent width limit measurements in regions where the residual is large, as can be the case with uncorrected telluric features or problematic sky subtraction. In cases such as these we select portions around the feature where the continuum estimate is reflected more accurately.

\section{A.2.4. Uncertainty in Central Wavelength}

If there is a known uncertainty in the wavelength center of the line, $\delta \lambda_{l}$, its effect on our equivalent width limits can be measured directly from the data. The variance of the equivalent width limit with respect to the wavelength center of the line is obtained by calculating the rest equivalent width limits over the range $\Delta \lambda_{i}= \pm \delta \lambda_{l}(1+z)$. Eq. A11 is used for the rest equivalent width limit calculations as before. The resulting variance is

$$
\sigma_{\lambda_{l}}^{2}\left(\sigma_{E W}\right)=\frac{1}{N_{\sigma_{E W}}-1} \sum_{n=\lambda_{i}^{-}}^{\lambda_{i}^{+}}\left(\sigma_{E W_{n}}-\left\langle\sigma_{E W}\right\rangle\right)^{2}
$$

where $\lambda_{i}^{+}$and $\lambda_{i}^{-}$are the upper and lower wavelengths set by $(1+z)\left(\lambda_{l} \pm \delta \lambda_{l}\right)$, respectively. $N_{\sigma_{E W}}$ is the total number of equivalent width limit calculations, and $\left\langle\sigma_{E W}\right\rangle$ is the mean equivalent width limit,

$$
\left\langle\sigma_{E W}\right\rangle=\frac{1}{N_{\sigma_{E W}}} \sum_{n=\lambda_{i}^{-}}^{\lambda_{i}^{+}} \sigma_{E W_{n}} .
$$

This technique more accurately reflects the errors due to uncertainties in wave center because it takes the actual data into account. If there is a problematic sky subtraction blended with the expected position of the feature the calculation will reflect this with a noticeably higher uncertainty. Also, if there is an uncertainty in the redshift, $z$, this can be incorporated into the calculation. 


\section{REFERENCES}

Bada, J. L. \& Lazcano, A. 2002, Science, 296, 1982

Bohlin, R. C., Savage, B. D., \& Drake, J. F. 1978, ApJ, 224, 132

Boissé, P., Le Brun, V., Bergeron, J., \& Deharveng, J. M. 1998, A\&A, 333, 841

Bouchet, P., Lequeux, J., Maurice, E., Prévot, L., \& Prévot-Burnichon, M. L. 1985, A\&A, 149, 330

Burbidge, E. M., Beaver, E. A., Cohen, R. D., Junkkarinen, V. T., \& Lyons, R. W. 1996, AJ, 112, 2533

Chen, H.-W. \& Lanzetta, K. M. 2003, ApJ, 597, 706

Chengalur, J. N., \& Kanekar, N., 1999, MNRAS, 302, L29

Cordiner, M. A., Cox, N. L. J., Trundle, C., Evans, C. J., Hunter, I., Przybilla, N., Bresolin, F., \& Salama, F. 2008, A\&A, 480, L13

Cox, N. L. J., Kaper, L., Foing, B. H., \& Ehrenfreund, P. 2005, A\&A, 438, 187

Cox, N. L. J., \& Spaans, M. 2006a, A\&A, 451, 973

Cox, N. L. J., Cordiner, M. A., Cami, J., Foing, B. H., Sarre, P. J., Kaper, L., \& Ehrenfreund, P. 2006b, A\&A, 447, 991

Cox, N. L. J., Cordiner, M. A., Ehrenfreund, P., Kaper, L., Sarre, P. J., Foing, B. H., Spaans, M., Cami, J., Sofia, U. J., Clayton, G. C., Gordon, K. D., \& Salama, F. 2007, A\&A, 470, 941

Dekker, H., D’Odorico, S., Kaufer, A. Delabre, B. \& Kotzlowski H. 2000, SPIE, 4008, 534

Ellison, S. L., Hall, P. B., \& Lira, P. 2005, AJ, 130, 1345

Ellison, S. L., York, B. A., Murphy, M. T., Zych, B. J., Smith, A. M., \& Sarre, P. J. 2008, MNRAS, 383, L30

Galazutdinov, G. A., Musaev, F. A., Krelowski, J. \& Walker, G. A. H. 2000, PASP, 112, 648

Gordon, K. D., Clayton, G. C., Misselt, K. A., Landolt, A. U., \& Wolff, M. J. 2003, ApJ, 594, 279

Heckman, T. M. \& Lehnert, M. D. 2000, ApJ, 537, 690

Heger, M. L. 1922, Lick Observatory Bull. 10, 337, 146

Herbig, G. H. 1993, ApJ, 407, 142

Herbig, G. H. 1995, ARA\&A, 33, 19 
Hobbs, L. M., York, D. G., Snow, T. P., Oka, T., Thorburn, J. A., Bishof, M., Friedman, S. D., McCall, B. J., Rachford, B., Sonnentrucker, P., \& Welty, D. E. 2007, ApJ, in preparation

Hudgins, D. M., Bauschlicher, C. W., Jr., \& Allamandola, L. J. 2005, ApJ, 632, 316

Jenniskens, P., \& Desert, F. -X. 1994, A\&A, 106, 39

Junkkarinen, V. T., Cohen, R. D., Beaver, E. A., Burbidge, E. M., \& Lyons, R. W. 2004, ApJ, 614, 658

Kanekar, N., \& Chengalur, J. N. 2001, A\&A, 369, 42

Khare P., Kulkarni, V. P., Lauroesch, J. T., York, D. G., Crotts, A. P. S. \& Nakamura, O. 2004, ApJ, 616, 86

Kulkarni, V. P., Fall, S. M., Lauroesch, J. T., York, D. G., Welty, D. E., Khare, P., \& Truran, J. W. 2005, ApJ, 618, 68

Lanzetta, K. M., Turnshek, D. A., \& Wolfe, A. M. 1987, ApJ, 322, 739

Le Brun, V., Bergeron, J., Boissé, P., \& Deharveng, J. M. 1997, A\&A, 321, 733

Lodders, K. 2003, ApJ, 591, 1220

Megier, A., Krelowski, J., \& Weselak, T. 2005, MNRAS, 358, 563

Ménard, B., Nestor, D., Turnshek, D., Quider, A., Richards, G., Chelouche, D., \& Rao, S. 2008, MNRAS, 385, 1053

Merrill, P. W. \& Wilson, O. C. 1938, ApJ, 87, 9

Moutou, C., Krelowski, J., d’Hendecourt, L., \& Jamroszczak, J. 1999, A\&A, 351, 680

Murphy, M. T. \& Liske, J. 2004, MNRAS, 354, L31

Murphy, M. T. 2006, UVES POPLER, http://www.ast.cam.ac.uk/ mim/UVES_popler.html

Rieke, G. H., Grasdalen, G. L., Kinman, T. D., Hintzen, P., Will, B. J., \& Wills, D. 1976, Nature, 260, 764

Rao, S. M. \& Turnshek, D. A. 1998, ApJ, 500, L115

Rao, S. M. \& Turnshek, D. A. 2000, ApJS, 130, 1

Rao, S. M., Nestor, D. B., Turnshek, D. A., Lane, W. M., Monier, E. M., \& Bergeron, J. 2003, ApJ, 595, 94

Rich, R. M. 1987, AJ, 94, 651 
Savage, B. D. \& Sembach, K. R. 1991, ApJ, 379, 245

Schneider, D. P., Hartig, G. F., Jannuzi, B. T., et al. 1993, ApJS, 87, 45

Sembach, K. R., Savage, B. D., \& Massa, D. 1991, ApJ, 372, 81

Snow, T. P. 2001, Spectrochimica Acta Part A, 57, 615

Sollerman, J., Cox, N., Mattila, S., Ehrenfreund, P., Kaper, L., Leibundgut, B., \& Lundqvist, P. 2005, A\&A, 429, 559

Steidel, C. C., Pettini, M., Dickinson, M., \& Persson, S. E. 1994, AJ, 108, 2046

Thorburn, J. A., Hobbs, L. M., McCall, B. J., Oka, T., Welty, D. E., Friedman, S. D., Snow, T. P., Sonnentrucker, P., \& York, D. G. 2003, ApJ, 584, 339

Tuairisg, S. O., Cami, J., Foing, B. H., Sonnentrucker, P., \& Ehrenfreund, P. 2000, A\&A, 142, 225

Turnshek, D. A., Rao, S. M., Nestor, D., Lane, W., Monier, E., Bergeron, J., \& Smette, A. 2001, ApJ, 553, 288

Turnshek, D. A., Rao, S. M., Ptak, A. F., Griffiths, R. E., \& Monier, E. M. 2003, ApJ, 590, 730

Welty, D. E., Federman, S. R., Gredel, R., Thorburn, J. A., \& Lambert, D. L. 2006, ApJS, 165, 138

Weselak, T., Schmidt, M., \& Krelowski, J. 2000, A\&A, 142, 239

Weselak, T., Fulara, J., Schmidt, M. R., \& Krelowski, J. 2001, A\&A, 377, 677

Wild, V. \& Hewett, P. C. 2005, MNRAS, 361, L30

Wild, V., Hewett, P. C., \& Pettini, M. 2006, MNRAS, 367, 211

Wszolek, B. \& Godlowski, W. 2003, MNRAS, 338, 990

Yanny, B., York, D. G., \& Gallagher, J. S. 1989, ApJ, 338, 735

Yan, L., Chary, R., Armus, L., Teplitz, H., Helou, G., Frayer, D., Fadda, D., Surace, J., \& Choi, P. 2005, ApJ, 628, 604

York, B. A., Ellison, S. L., Lawton, B., Churchill, C. W., Snow, T. P., Johnson, R. A., \& Ryan, S. G. 2006, ApJ, 647, L29 
Table 1. DLAs in Sample

\begin{tabular}{|c|c|c|c|c|c|c|c|}
\hline DLA & & QSO & $z_{a b s}$ & $\begin{array}{c}N(\mathrm{HI}) / 10^{20} \\
{[\text { atoms cm }} \\
-2\end{array}$ & {$[\mathrm{Zn} / \mathrm{H}]$} & {$[\mathrm{Fe} / \mathrm{H}]$} & Refs. \\
\hline 1 & $\mathrm{AO}$ & $0235+164^{a}$ & 0.524 & $50 \pm 10$ & $\ldots$ & $\cdots$ & 1,2 \\
\hline 2 & $\mathrm{Q}$ & $0738+313$ & 0.091 & $15 \pm 2$ & $<-1.14$ & $-1.63_{-0.18}^{+0.13}$ & $3,4,8,9$ \\
\hline 3 & & & 0.221 & $7.9 \pm 1.4$ & $<-0.70_{-0.17}^{+0.14}$ & $\cdots$ & $3,4,8,9$ \\
\hline 4 & $\mathrm{~B} 2$ & $0827+243$ & 0.518 & $2.0 \pm 0.2$ & $<+0.30$ & $-1.02 \pm 0.05$ & $4,5,8,9$ \\
\hline 5 & PKS & $0952+179$ & 0.239 & $21 \pm 3$ & $<-1.02$ & $\cdots$ & $4,5,9$ \\
\hline 6 & PKS & $1127-145$ & 0.313 & $51 \pm 9$ & $\cdots$ & $>-2$ & $1,6,9$ \\
\hline 7 & $\mathrm{Q}$ & $1229-020$ & 0.395 & $5.6 \pm 1.0$ & -0.47 & $<-1.32$ & 7 \\
\hline
\end{tabular}

Note. - All upper limits are calculated to $3 \sigma$.

${ }^{a}$ Metallicity for AO $0235+164$ is estimated via X-ray spectroscopy to be $Z=0.24 \pm 0.06 Z_{\odot}($ Chan$d r a$, Turnshek et al. 2003) and $Z=0.72 \pm 0.28 Z_{\odot}$ (ASCA \& ROSAT, Junkkarinen et al. 2004).

References. - 1. Turnshek et al. (2003); 2. Junkkarinen et al. (2004); 3. Rao \& Turnshek (1998); 4. Kulkarni et al. (2005); 5. Rao \& Turnshek (2000); 6. This work; 7. Boissé et al. (1998); 8. Khare et al. (2004); 9. Kanekar \& Chengalur (2003) 
Table 2. Journal of Observations

\begin{tabular}{|c|c|c|c|c|c|c|c|c|c|c|}
\hline & QSO & Facility & $\begin{array}{l}\text { Grating/ } \\
\text { Grism }\end{array}$ & $\begin{array}{c}\text { Slit } \\
\text { Width }\end{array}$ & Date $[\mathrm{UT}]$ & $\begin{array}{c}\text { Exposure } \\
\text { Time }[\mathrm{s}]\end{array}$ & $\begin{array}{c}\mathrm{S} / \mathrm{N} \\
{\left[\mathrm{pixel}^{-1}\right]}\end{array}$ & $\begin{array}{c}\text { Wavelength } \\
\text { Coverage }[\AA]\end{array}$ & Resolution & $\begin{array}{c}\text { Dispersion } \\
{\left[\AA \text { pixel }^{-1}\right]}\end{array}$ \\
\hline $\mathrm{AO}$ & $0235+164$ & VLT/FORS2 & GRIS 600z & $1.0 "$ & $\begin{array}{l}2005 \text { Jul } 20 \\
2005 \text { Sep } 6 \\
2005 \text { Oct } 1\end{array}$ & 8400 & $60-150$ & $7318-10,744$ & 1880 & 1.59 \\
\hline Q & $0738+313$ & APO/DIS & HIGH & $1.5 "$ & $\begin{array}{l}2004 \text { Dec } 15 / 19 \\
2005 \text { Feb } 4\end{array}$ & 40,600 & $57-84$ & $4367-7817$ & 2040 & $\begin{array}{c}0.62 / 0.84 \\
\text { (Blue/Red) }\end{array}$ \\
\hline B2 & $0827+243$ & Keck/HIRES & & $\mathrm{C} 2 / 0.861 "$ & 1998 Dec 22 & 22,500 & $27-114^{a}$ & 5185-9234 & 43,000 & 0.04 \\
\hline PKS & $0952+179$ & VLT/FORS2 & GRIS 600RI & $1.0 "$ & 2005 Apr $4 / 5 / 7 / 8$ & 9000 & $57-95$ & $5298-8622$ & 1650 & 1.63 \\
\hline PKS & $0952+179$ & WHT/ISIS & R600R & $1.0 "$ & 2004 May 20 & 4500 & $20-22$ & $6312-8114$ & 3790 & 0.45 \\
\hline PKS & $1127-145$ & VLT/UVES & 346,564 & $1.0 "$ & 2002 Jul $17 / 18$ & 24,900 & $38^{b}$ & $3041-6809$ & 45,000 & 0.05 \\
\hline PKS & $1127-145$ & Gemini/GMOS-S & $\mathrm{R} 400$ & $1.0 "$ & 2004 Jun 19 & 3600 & $38-84$ & 5962-9998 & 960 & 2.75 \\
\hline Q & $1229-020$ & VLT/FORS2 & GRIS 600z & $1.0 "$ & 2005 Apr $9 / 13$ & 9800 & $50-62$ & $7464-10,000$ & 1860 & 1.59 \\
\hline Q & $1229-020$ & WHT/ISIS & R600R & $1.0 "$ & 2004 May 20 & 3600 & $5-13$ & $7212-9018$ & 4980 & 0.45 \\
\hline
\end{tabular}

${ }^{a}$ The low $\mathrm{S} / \mathrm{N}$ is measured in the red, and the high $\mathrm{S} / \mathrm{N}$ is measured in the blue.

${ }^{b}$ The $\mathrm{S} / \mathrm{N}$ is measured at the location of the $\lambda 4428$ DIB. The other DIBs are not covered in this spectrum. 
Table 3. Equivalent Width Detections \& Limits of DIBs in DLAs

\begin{tabular}{|c|c|c|c|c|c|c|c|c|c|c|}
\hline \multirow[b]{2}{*}{ DLA } & & \multirow[b]{2}{*}{ QSO } & \multirow[b]{2}{*}{$z_{a b s}$} & \multirow[b]{2}{*}{ Facility } & \multicolumn{6}{|c|}{ Rest-Frame EW Detections \& Rest-Frame $3 \sigma$ EW Limits $[\mathrm{m} \AA]$} \\
\hline & & & & & $\lambda 4428$ & $\lambda 5705$ & $\lambda 5780$ & $\lambda 5797$ & $\lambda 6284$ & $\lambda 6613$ \\
\hline 1 & $\mathrm{AO}$ & $0235+164$ & 0.524 & VLT/FORS2 & $741.5 \pm 26.2^{a}$ & $63.2 \pm 8.7^{b}$ & $216 \pm 9^{b}$ & $<118^{b c}$ & $<128^{b c}$ & $<95^{b c}$ \\
\hline 2 & Q & $0738+313$ & 0.091 & APO/DIS & $<115(8)$ & $\ldots$ & $<88(6)^{c}$ & $<74(3)$ & $<106(3)$ & $<414(51)$ \\
\hline 3 & & & 0.221 & APO/DIS & $<151(11)$ & $\ldots$ & $<85(3)$ & $<78(3)$ & $<240(16)^{c}$ & $\ldots$ \\
\hline 4 & $\mathrm{~B} 2$ & $0827+243$ & 0.518 & Keck/HIRES & $<94(14)^{a}$ & $\ldots$ & $<32(1)$ & $<21(1)$ & $\ldots$ & $\ldots$ \\
\hline \multirow[t]{2}{*}{5} & PKS & $0952+179$ & 0.239 & VLT/FORS2 & $<259(19)$ & $\ldots$ & $<90(4)$ & $<289(14)^{c}$ & $<102(4)$ & $<108(6)$ \\
\hline & & & 0.239 & WHT/ISIS & .. & $\ldots$ & $<158(4)$ & $<237(18)$ & $<187(3)$ & .. \\
\hline \multirow[t]{2}{*}{6} & PKS & $1127-145$ & 0.313 & VLT/UVES & $<68(5)^{d}$ & $\ldots$ & $\ldots$ & ... & ... & $\ldots$ \\
\hline & & & 0.313 & Gemini/GMOS & $\ldots$ & $\ldots$ & $<6106(77)^{c d}$ & $<3162(116)^{c d}$ & $<341(1)^{d}$ & $<380(1)^{d}$ \\
\hline \multirow[t]{2}{*}{7} & $\mathrm{Q}$ & $1229-020$ & 0.395 & VLT/FORS2 & $\ldots$ & $\ldots$ & $<129(5)$ & $<115(6)$ & $<162(5)$ & $<135(7)$ \\
\hline & & & 0.395 & WHT/ISIS & $\cdots$ & $\cdots$ & $<292(10)$ & $<198(8)$ & $<627(14)^{c}$ & $\cdots$ \\
\hline
\end{tabular}

${ }^{a}$ Spectrum provided by Junkkarinen et al. (2004).

${ }^{b}$ Spectrum provided by York et al. (2006).

${ }^{c}$ Problematic sky subtraction or large atmospheric band.

${ }^{d}$ Uncertainty in resolution is unknown and not included in error assessment. 
Table 4. Model Predictions of DIBs in DLAs

\begin{tabular}{|c|c|c|c|c|c|c|c|c|}
\hline \multirow[b]{2}{*}{ DLA } & & \multirow{2}{*}{$\begin{array}{l}\mathrm{QSO} \\
z_{\mathrm{DLA}}\end{array}$} & \multirow{2}{*}{$\begin{array}{l}\text { Observed/ } \\
\text { Models }^{c}\end{array}$} & \multirow{2}{*}{$\begin{array}{c}\text { Rest-Frame V } \\
\quad \lambda 4428\end{array}$} & \multirow{2}{*}{$\begin{array}{c}\mathrm{V} \text { Detections, } \\
\lambda 5780\end{array}$} & \multirow{2}{*}{$\begin{array}{c}\text { Limits }(3 \sigma), \& \\
\lambda 5797\end{array}$} & \multicolumn{2}{|c|}{ Model Predictions $[\mathrm{mA}]$} \\
\hline & & & & & & & $\lambda 6284$ & $\lambda 6613$ \\
\hline \multirow[t]{4}{*}{1} & $\mathrm{AO}$ & $0235+164$ & Observed & $741.5 \pm 26.2^{a}$ & $216 \pm 9^{b}$ & $<118^{b}$ & $<128^{b}$ & $<95^{b}$ \\
\hline & & $z=0.524$ & $\mathrm{EW}[N(\mathrm{HI})]$ & $\cdots$ & 1059 & 317 & 1572 & $\cdots$ \\
\hline & & & $\mathrm{EW}[E(B-V)=0.23]$ & 482 & 104 & 43 & 368 & 50 \\
\hline & & & $E(B-V)_{\lim }$ & 0.35 & 0.48 & 0.64 & 0.06 & 0.44 \\
\hline \multirow[t]{4}{*}{2} & $\mathrm{Q}$ & $0738+313$ & Observed & $<115(8)$ & $<88(6)$ & $<74(3)$ & $<106(3)$ & $<414(51)$ \\
\hline & & $z=0.091$ & $\mathrm{EW}[N(\mathrm{HI})]$ & $\cdots$ & 247 & 89 & 532 & $\cdots$ \\
\hline & & & $\mathrm{EW}[E(B-V)=0.04]$ & 84 & 18 & 8 & 91 & 3 \\
\hline & & & $E(B-V)_{\lim }$ & 0.05 & 0.20 & 0.40 & 0.05 & 1.91 \\
\hline \multirow[t]{4}{*}{3} & $\mathrm{Q}$ & $0738+313$ & Observed & $<151(11)$ & $<85(3)$ & $<78(3)$ & $<240(16)$ & $\cdots$ \\
\hline & & $z=0.221$ & $\mathrm{EW}[N(\mathrm{HI})]$ & $\cdots$ & 114 & 46 & 299 & $\cdots$ \\
\hline & & & $\mathrm{EW}[E(B-V)=0.04]$ & 84 & 18 & 8 & 91 & $\cdots$ \\
\hline & & & $E(B-V)_{\lim }$ & 0.07 & 0.19 & 0.42 & 0.14 & $\cdots$ \\
\hline \multirow[t]{4}{*}{4} & B2 & $0827+243$ & Observed & $<94(14)$ & $<32(1)$ & $<21(1)$ & $\cdots$ & $\cdots$ \\
\hline & & $z=0.518$ & $\mathrm{EW}[N(\mathrm{HI})]$ & $\ldots$ & 22 & 11 & $\cdots$ & $\cdots$ \\
\hline & & & $\mathrm{EW}[E(B-V)=0.04]$ & 84 & 18 & 8 & $\cdots$ & $\cdots$ \\
\hline & & & $E(B-V)_{\lim }$ & 0.04 & 0.07 & 0.11 & $\cdots$ & $\cdots$ \\
\hline \multirow[t]{4}{*}{5} & PKS & $0952+179$ & Observed & $<259(19)$ & $<90(4)$ & $<237(18)$ & $<102(4)$ & $<108(6)$ \\
\hline & & $z=0.239$ & $\mathrm{EW}[N(\mathrm{HI})]$ & $\ldots$ & 370 & 127 & 720 & $\ldots$ \\
\hline & & & $\mathrm{EW}[E(B-V)=0.04]$ & 84 & 18 & 8 & 91 & 3 \\
\hline & & & $E(B-V)_{\lim }$ & 0.12 & 0.20 & 1.29 & 0.05 & 0.50 \\
\hline \multirow[t]{4}{*}{6} & PKS & $1127-145$ & Observed & $<68(5)$ & $<6106(77)$ & $<3162(116)$ & $<341(1)$ & $<380(1)$ \\
\hline & & $z=0.313$ & $\mathrm{EW}[N(\mathrm{HI})]$ & $\cdots$ & 1086 & 324 & 1601 & $\ldots$ \\
\hline & & & $\mathrm{EW}[E(B-V)=0.04]$ & 84 & 18 & 8 & 91 & 3 \\
\hline & & & $E(B-V)_{\lim }$ & 0.03 & 14.13 & 17.73 & 0.21 & 1.75 \\
\hline \multirow[t]{4}{*}{7} & $\mathrm{Q}$ & $1229-020$ & Observed & $\cdots$ & $<129(5)$ & $<115(6)$ & $<162(5)$ & $<135(7)$ \\
\hline & & $z=0.395$ & $\mathrm{EW}[N(\mathrm{HI})]$ & $\cdots$ & 75 & 32 & 219 & $\cdots$ \\
\hline & & & $\mathrm{EW}[E(B-V)=0.04]$ & $\cdots$ & 18 & 8 & 91 & 3 \\
\hline & & & $E(B-V)_{\lim }$ & $\cdots$ & 0.29 & 0.62 & 0.08 & 0.62 \\
\hline
\end{tabular}

${ }^{a}$ Spectrum provided by Junkkarinen et al. (2004).

${ }^{b}$ Spectrum provided by York et al. (2006).

${ }^{c}$ The limits are those that are the best constrained from Table 3 The model $\mathrm{EW}[N(\mathrm{HI})]$ refers to the predicted equivalent widths $(\mathrm{m} \AA)$ from the Galactic best-fit lines in Figure 9 The values used for the $N(\mathrm{HI})$ are taken from Table 1 The model $\mathrm{EW}[E(B-V)=0.23]$ refers to the predicted equivalent widths $(\mathrm{m} \AA)$ from the Galactic DIB- $E(B-V)$ best-fit lines with the known $E(B-V)=0.23$ (Junkkarinen et al. 2004). The model $\mathrm{EW}[E(B-V)=0.04]$ refers to the predicted equivalent widths $(\mathrm{m} \AA)$ from the Galactic DIB- $E(B-V)$ best-fit lines with the upper limit of $E(B-V)=0.04$ as measured in high-z DLAs by Ellison et al. (2005). For all systems, the $E(B-V)=0.04$ except for AO $0235+164$ which has a measured $E(B-V)=0.23$ (Junkkarinen et al. 2004). The value $E(B-V)_{\text {lim }}$ is the $E(B-V)$ upper limit inferred from our equivalent width limits and the Galactic DIB $-E(B-V)$ best-fit lines for the $\lambda 5780, \lambda 5797$, and $\lambda 6284$ DIBs (Welty et al. 2006) and the $\lambda 4428$ and $\lambda 6613$ DIBs (unpublished, T.P. Snow, private communication). 
Table 5. Adopted Reddening and Gas-to-Dust Ratios

\begin{tabular}{rrllcrc}
\hline \hline DLA & & QSO & $z_{a b s}$ & $\begin{array}{c}E(B-V) \\
{[\mathrm{mag}]}\end{array}$ & $\begin{array}{c}\text { gas/dust } \\
{\left[\mathrm{cm}^{-2} \mathrm{mag}^{-1}\right]}\end{array}$ & $\begin{array}{c}\text { Contraint } \\
\text { DIB }\end{array}$ \\
\hline 1 & AO & $0235+164$ & $0.524^{a}$ & 0.23 & $19.2 \times 10^{21}$ & $\ldots$ \\
2 & $\mathrm{Q}$ & $0738+313$ & 0.091 & $<0.05$ & $>30 \times 10^{21}$ & $\lambda 6284$ \\
3 & & & 0.221 & $<0.14$ & $>5.6 \times 10^{21}$ & $\lambda 6284$ \\
4 & $\mathrm{~B} 2$ & $0827+243$ & 0.518 & $<0.07$ & $>2.9 \times 10^{21}$ & $\lambda 5780$ \\
5 & $\mathrm{PKS}$ & $0952+179$ & 0.239 & $<0.05$ & $>42 \times 10^{21}$ & $\lambda 6284$ \\
6 & $\mathrm{PKS}$ & $1127-145$ & 0.313 & $<0.21$ & $>24 \times 10^{21}$ & $\lambda 6284$ \\
7 & $\mathrm{Q}$ & $1229-020$ & 0.395 & $<0.08$ & $>7.0 \times 10^{21}$ & $\lambda 6284$ \\
\hline
\end{tabular}

${ }^{a}$ Values quoted from Junkkarinen et al. (2004). 


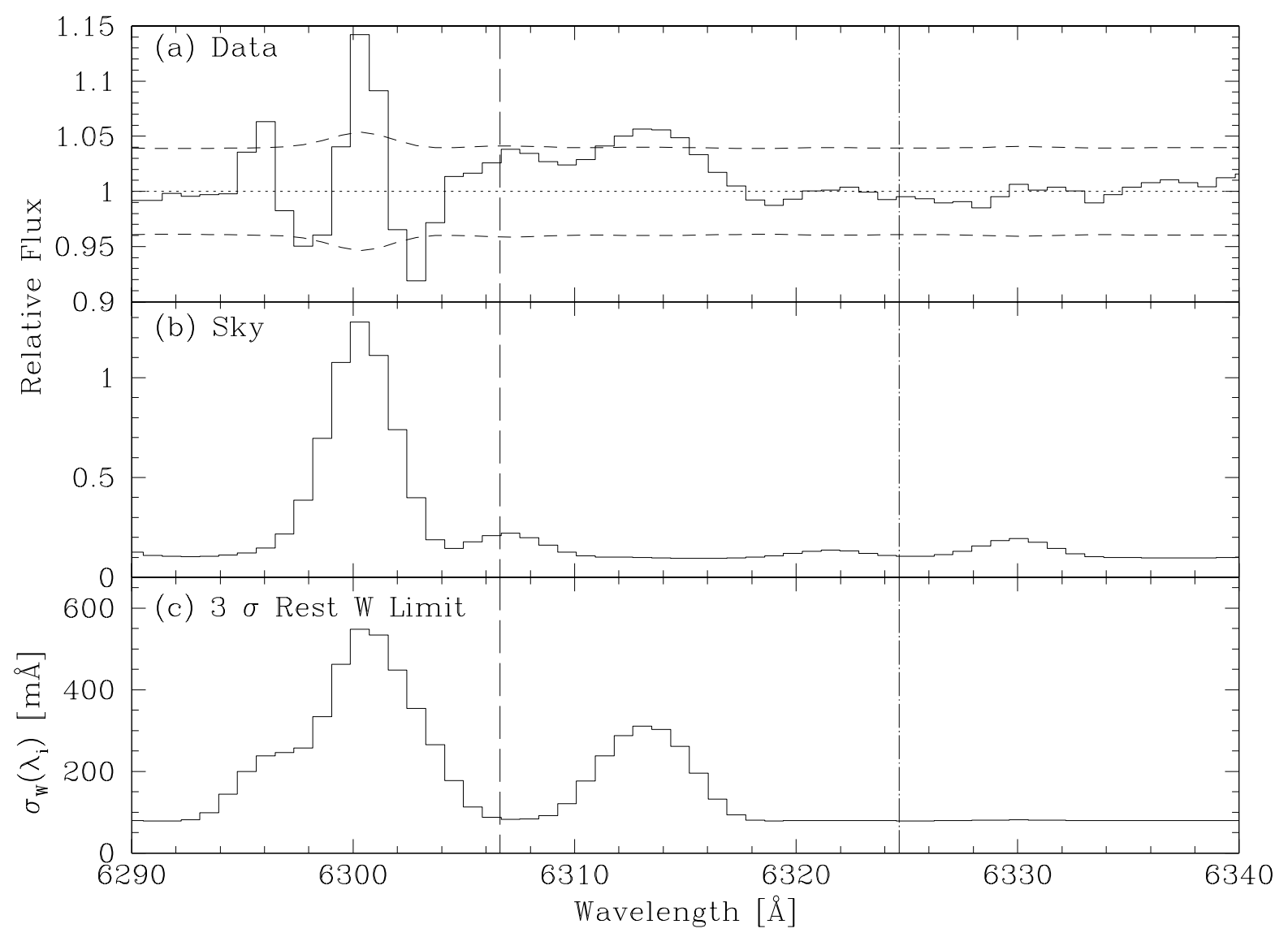

Fig. 1. - Equivalent width limit analysis of the Q0738+313 $z=0.091$ DLA. - (a) Relative flux in the region of the $\lambda 5780$ and $\lambda 5797$ DIBs (vertical long dashed line and vertical long dashed-dotted line, respectively). The histogram is the relative flux data, and the short dashed lines are the associated $\pm 3 \sigma$ flux errors. - $(b)$ Relative sky flux. - $-(c)$ The $3 \sigma$ rest equivalent width limits in $\mathrm{m} \AA$. The two peaks in panel $(c)$ are due to the problematic sky subtraction from the [OI] sky emission line at $\lambda \sim 6300 \AA$ and the poor continuum fit at $\lambda \sim 6314 \AA$ where the residuals are used, as explained in Eq. A8. 

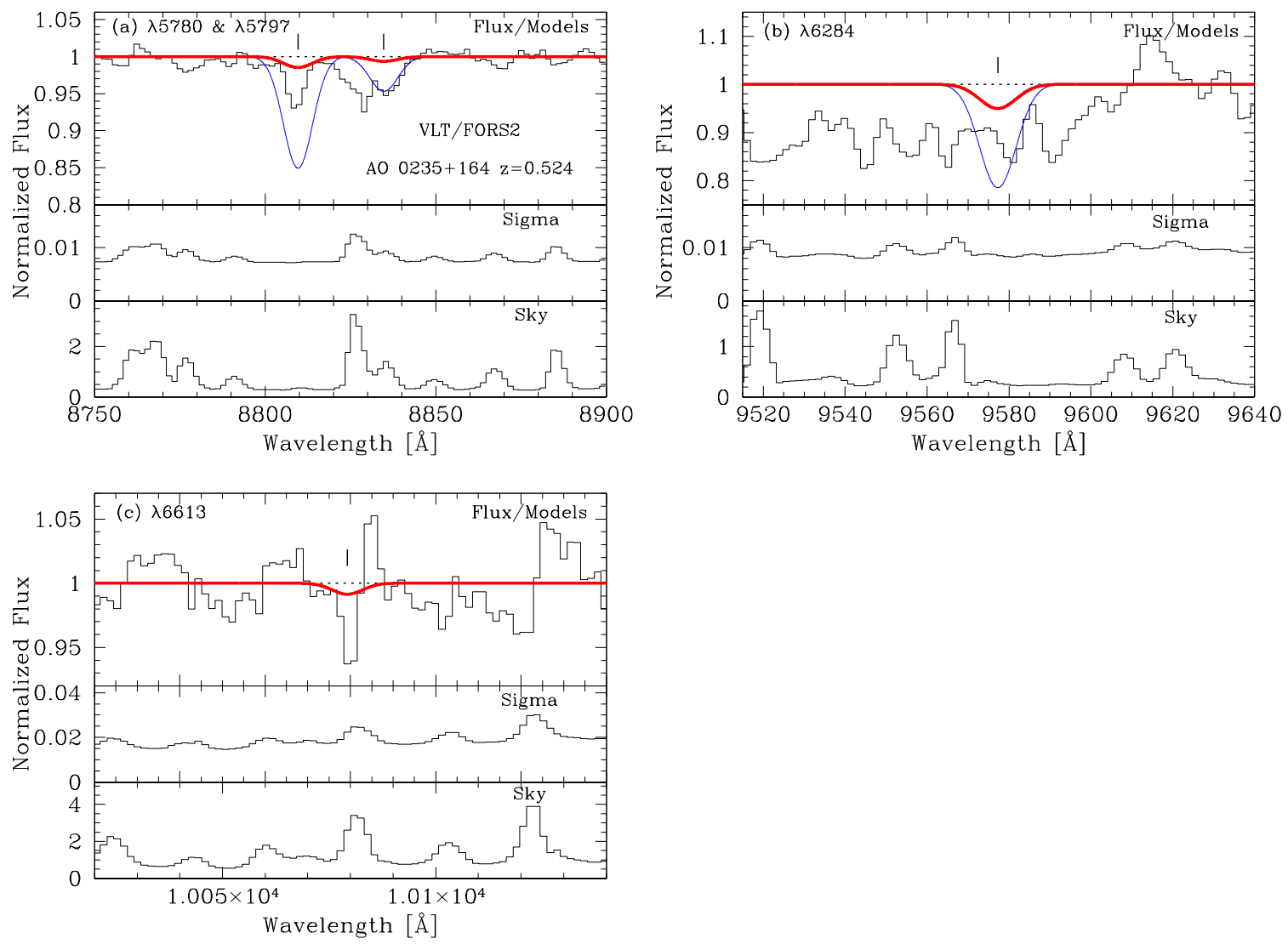

Fig. 2. - VLT/FORS2 spectrum of the $z=0.524$ DLA toward AO 0235+164. Plotted are the expected positions of the (a) $\lambda 5780 / 5797,(b) \lambda 6284$, and (c) $\lambda 6613$ DIBs. The upper sub-panels are the normalized flux of the data and models. The center sub-panels are the sigmas of the associated data fluxes normalized by the continuum. The lower sub-panels are the sky fluxes normalized by the continuum. The smooth curves are model predictions (see text). The thin curves are the expected DIB profiles given the measured DLA $N(\mathrm{HI})$ and the Galactic DIB- $N(\mathrm{HI})$ relation. The thick curves are the expected DIB profiles using the measured $E(B-V)=0.23$ from Junkkarinen et al. (2004). 

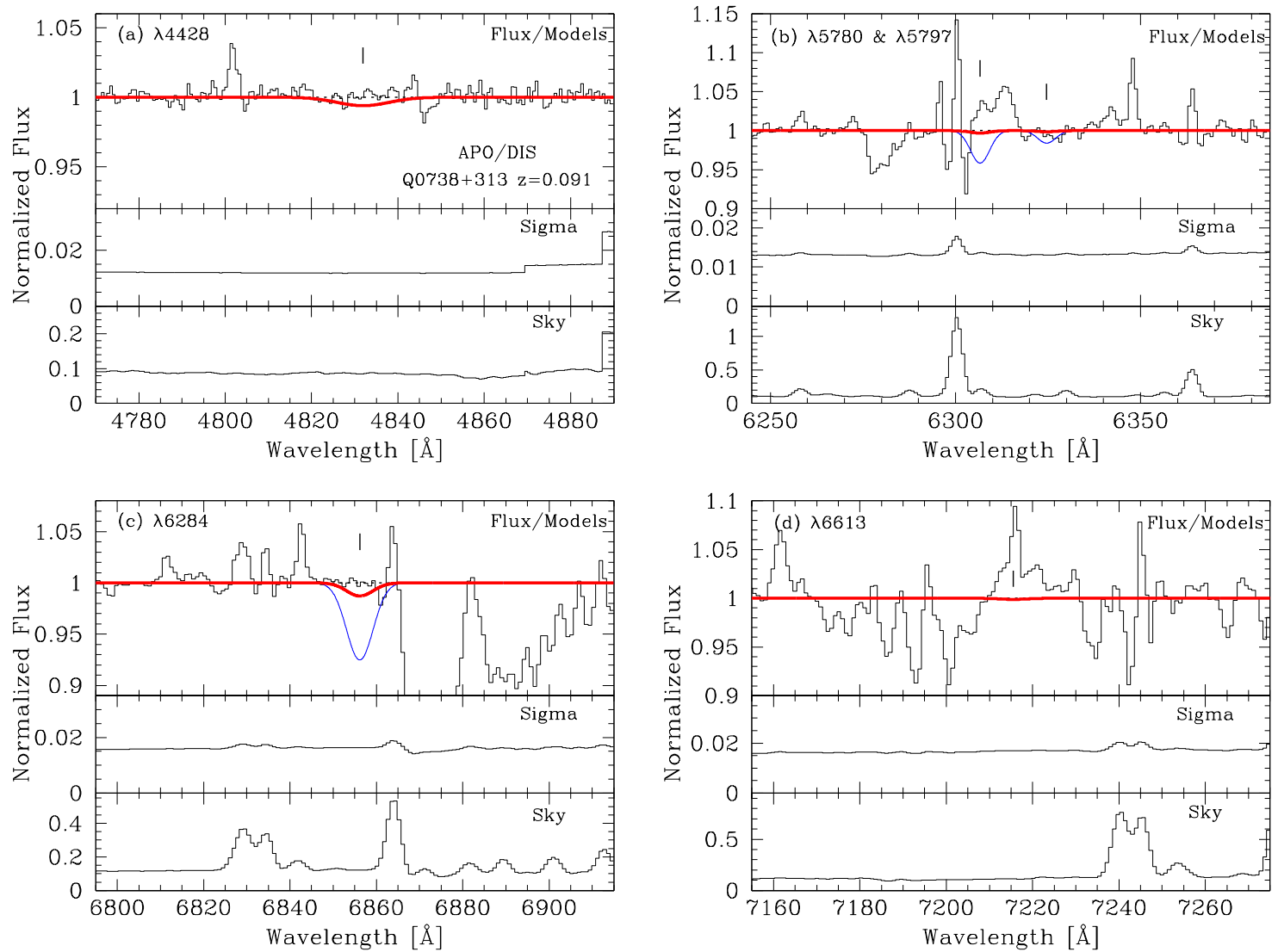

Fig. 3.- Same as Fig. 2, but for the APO/DIS spectrum of the $z=0.091$ DLA toward Q0738+313. Plotted are the expected positions of the $(a) \lambda 4428,(b) \lambda 5780 / 5797,(c) \lambda 6284$, and $(d) \lambda 6613$ DIBs. The smooth curves are model predictions (see text). The thin curves are the expected DIB profiles given the measured DLA $N(\mathrm{HI})$ and the Galactic DIB- $N(\mathrm{HI})$ relation. The thick curves for this DLA are the expected DIB profiles using the $E(B-V)=0.04$ upper limit for high-z DLAs from Ellison et al. (2005). 

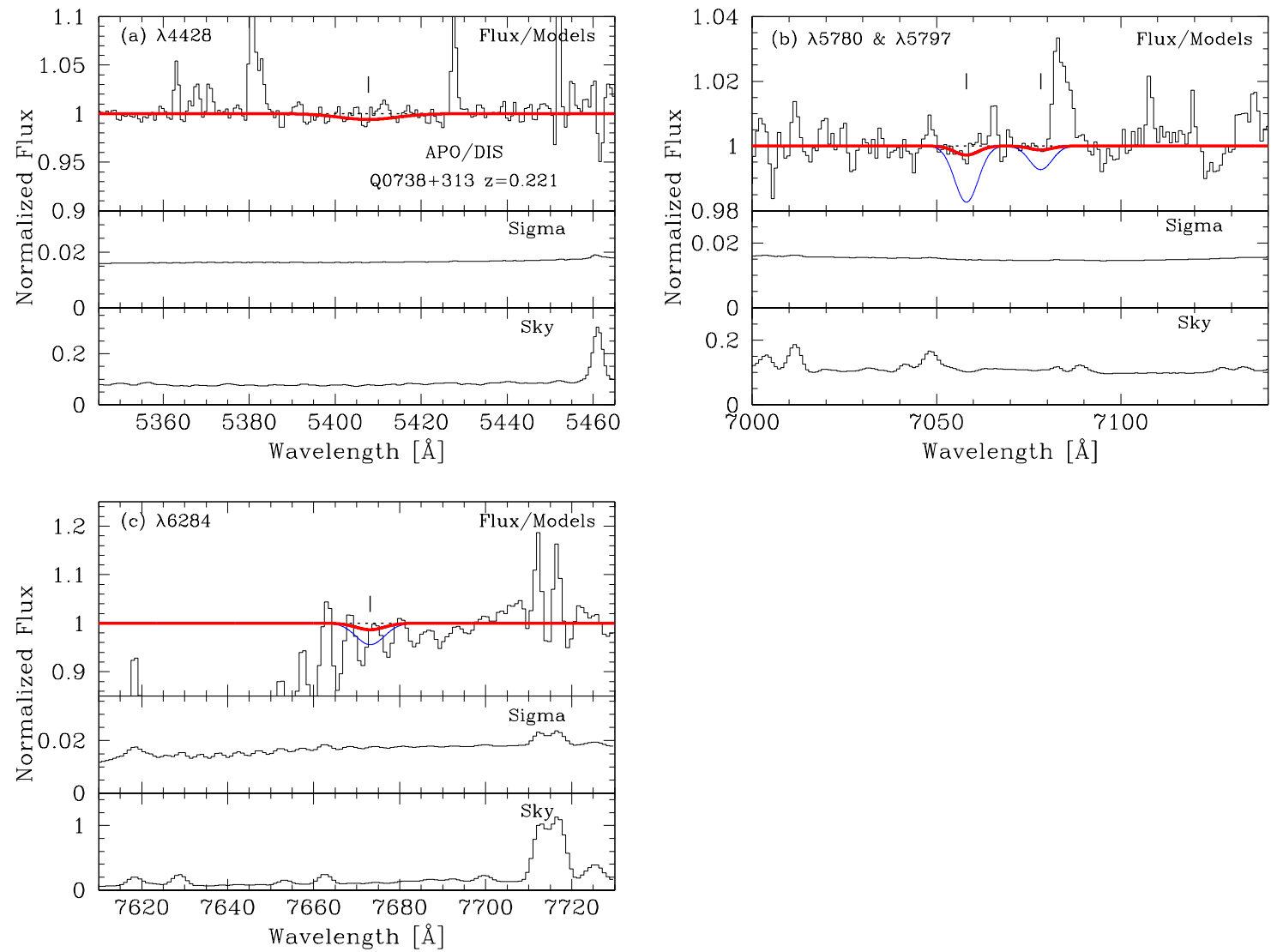

Fig. 4. - Same as Fig. 3, but for the APO/DIS spectrum of the $z=0.221$ DLA toward Q0738+313. Plotted are the expected positions of the $(a) \lambda 4428,(b) \lambda 5780 / 5797$, and (c) $\lambda 6284$ DIBs. 

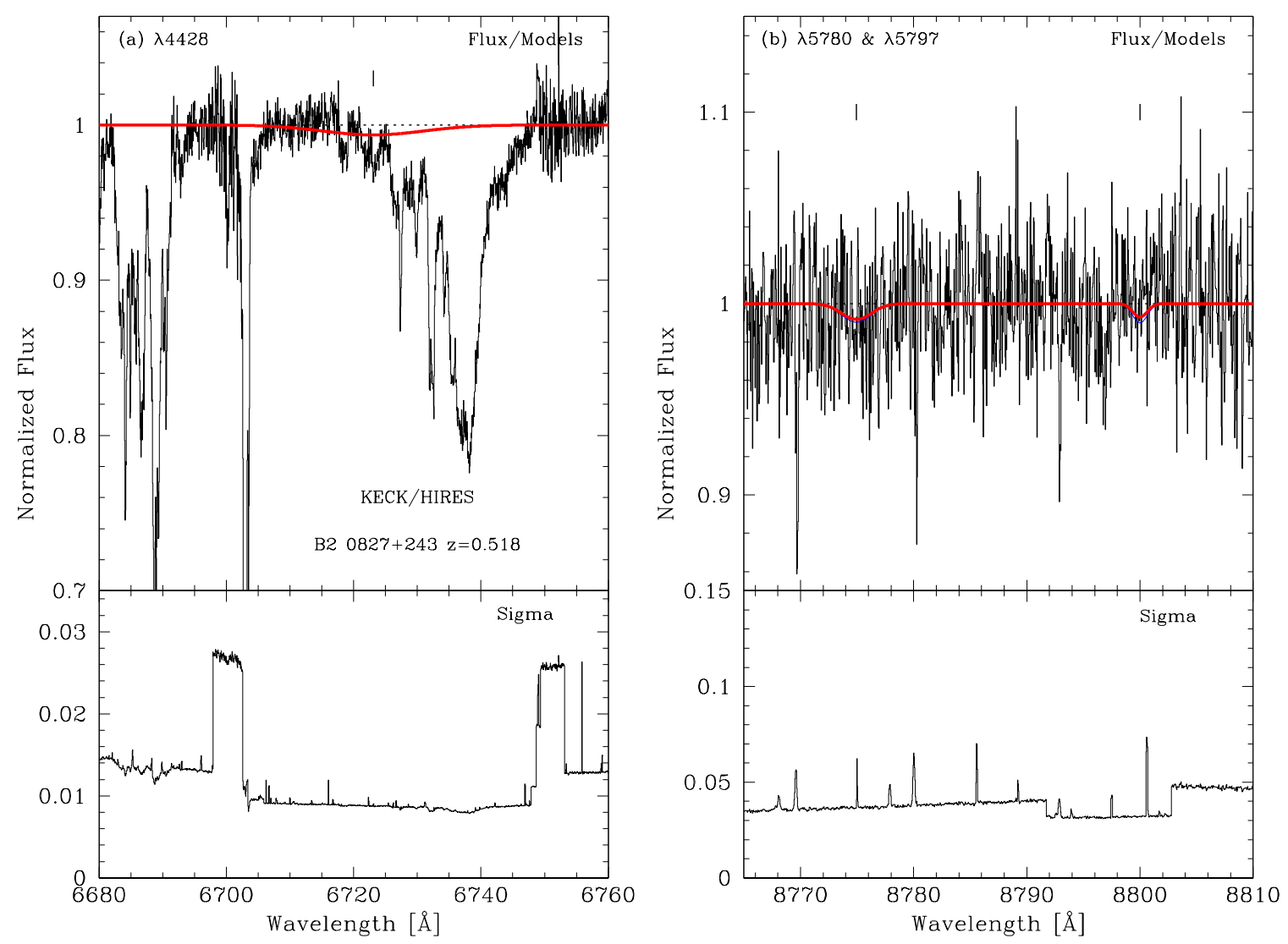

Fig. 5.- Same as Fig. 3, but for the Keck/HIRES spectrum of the $z=0.518$ DLA toward B2 $0827+243$. Plotted are the expected positions of the (a) $\lambda 4428$ and the $(b) \lambda 5780 / 5797$ DIBs. 

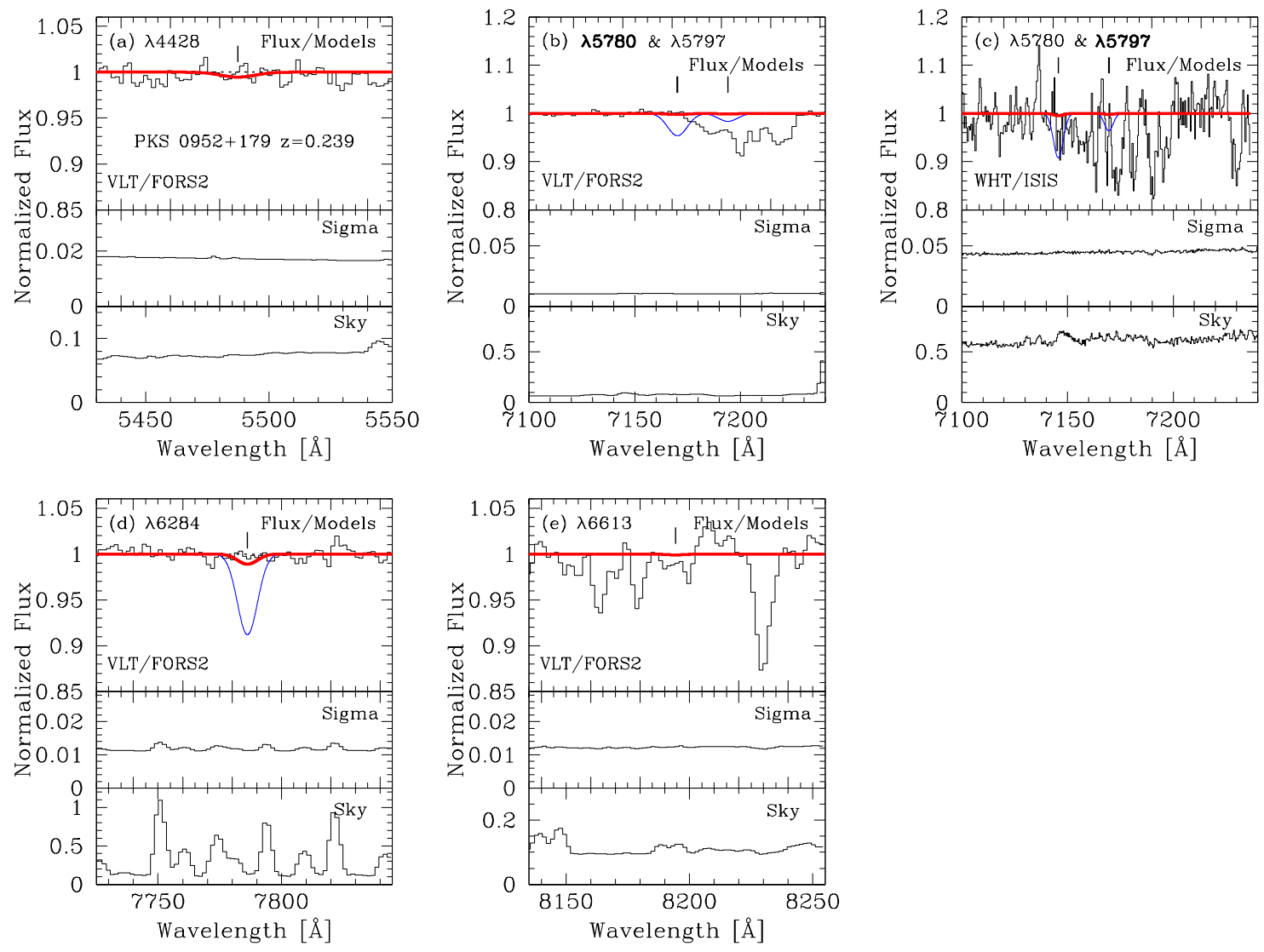

Fig. 6. - Same as Fig. 3, but for the VLT/FORS2 and WHT/ISIS spectra of the $z=0.239$ DLA toward PKS 0952+179. Plotted are the expected positions of the $(a) \lambda 4428,(b) \lambda 5780 / 5797,(c)$ $\lambda 5780 / 5797$ (WHT/ISIS), (d) $\lambda 6284$, and (e) $\lambda 6613$ DIBs. The WHT/ISIS spectrum provides the adopted limit for the $\lambda 5797$ DIB; the VLT/FORS2 spectrum provides the adopted limits for the remaining DIBs. 

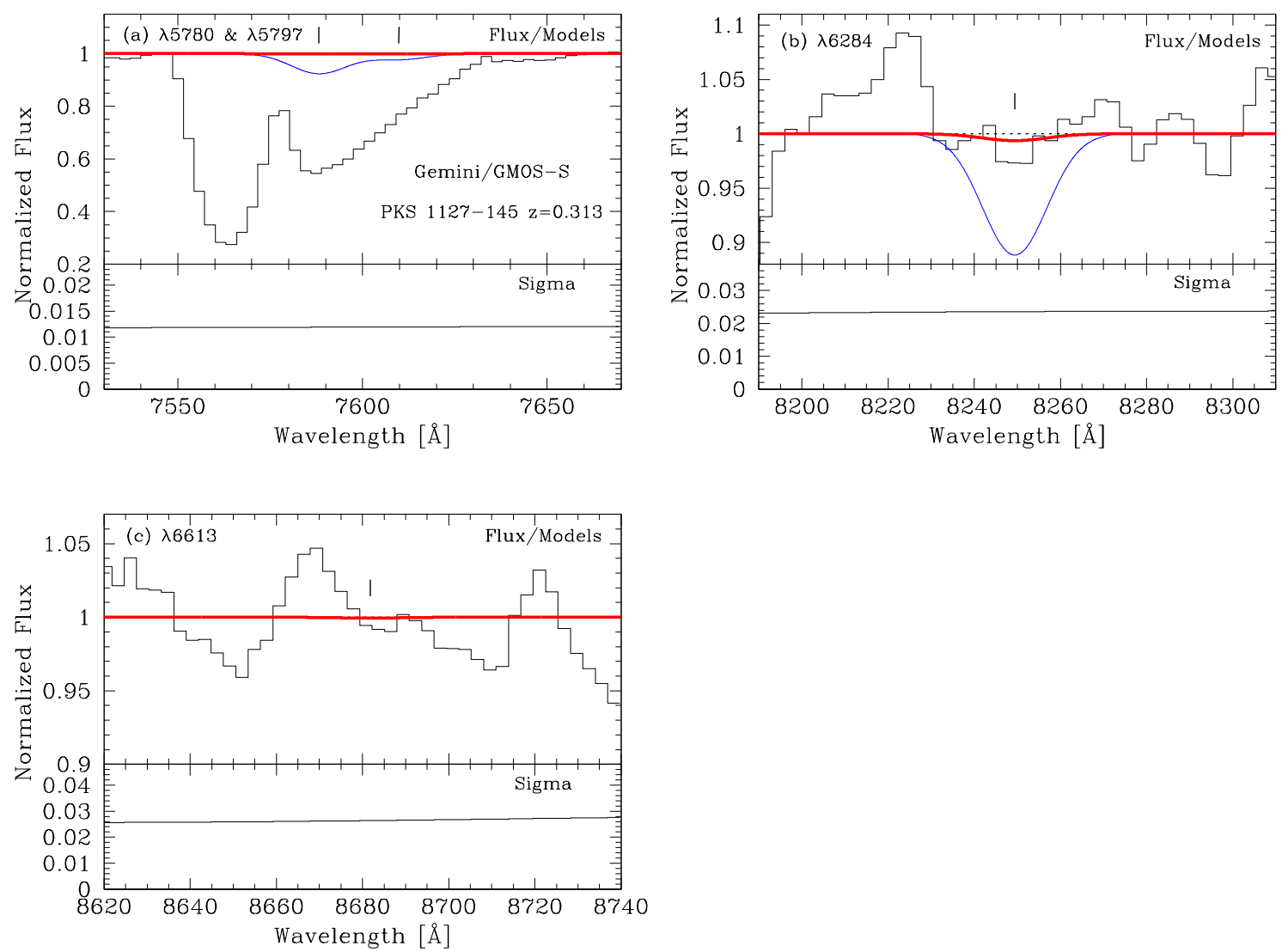

Fig. 7.- Same as Fig. 3, but for the Gemini/GMOS-S spectrum of the $z=0.313$ DLA toward PKS 1127-145. Plotted are the expected positions of the (a) $\lambda 5780 / 5797,(b) \lambda 6284$, and (c) $\lambda 6613$ DIBs. 

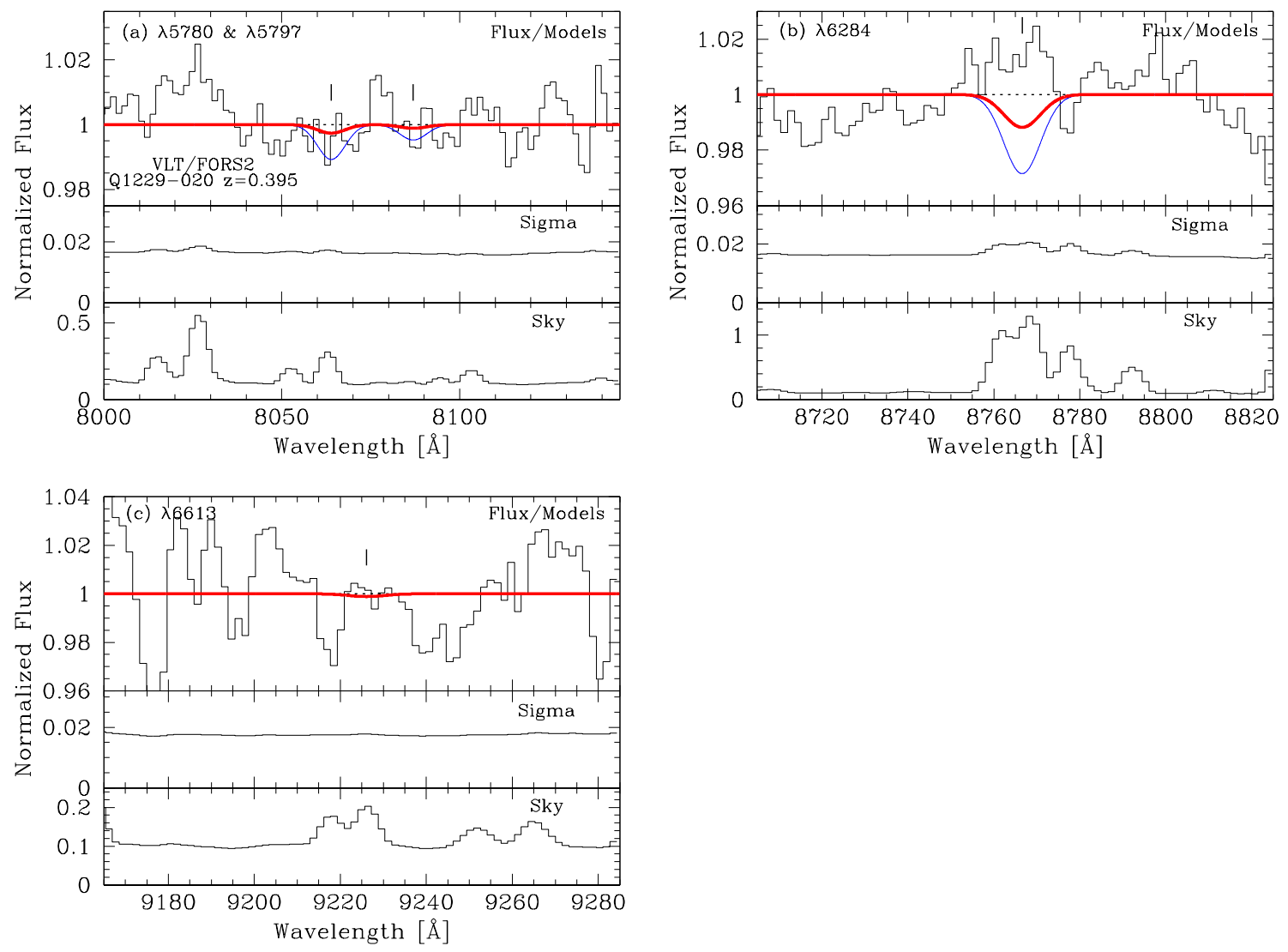

Fig. 8. - Same as Fig. 3, but for the VLT/FORS2 spectrum of the $z=0.395$ DLA toward Q1229020. Plotted are the expected positions of the $(a) \lambda 5780 / 5797,(b) \lambda 6284$, and (c) $\lambda 6613$ DIBs. 


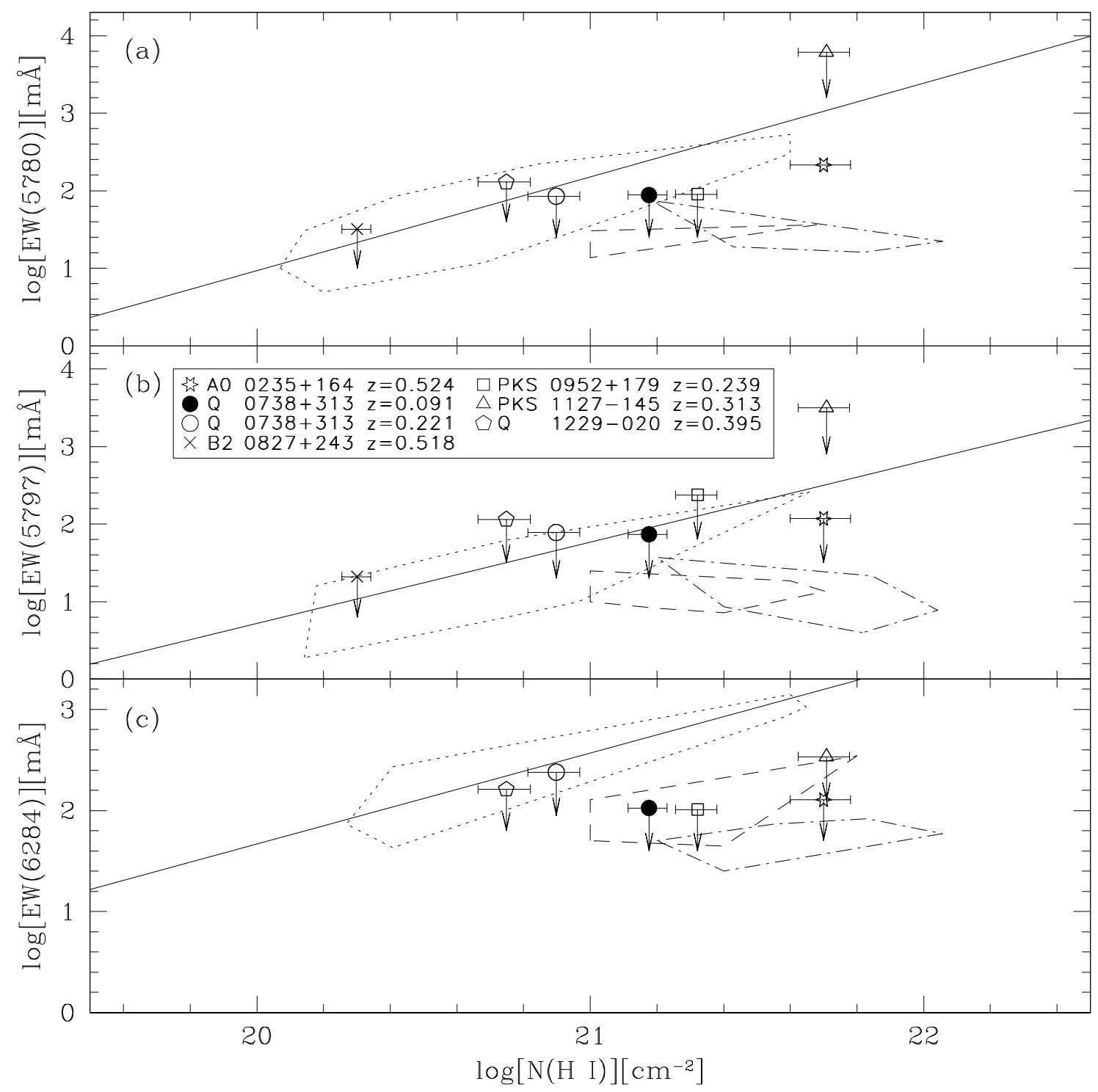

Fig. 9. - The DIB equivalent width-N(HI) relations (Welty et al. 2006) with our DLAs added. - (a) $\lambda 5780$ DIB. - (b) $\lambda 5797$ DIB. - (c) $\lambda 6284$ DIB. The solid lines are the best-fit weighted Galactic lines. The region enclosed by the dotted lines contains the Galactic data. The region enclosed by the dashed lines contains the LMC data. The region enclosed by the dot-dash lines contains the SMC data. Error bars are $1 \sigma$, and upper limits are marked with arrows. The vertical error bars for AO 0235+164 in panel $(a)$ are smaller than the point size and all values for this DLA are from York et al. (2006). 


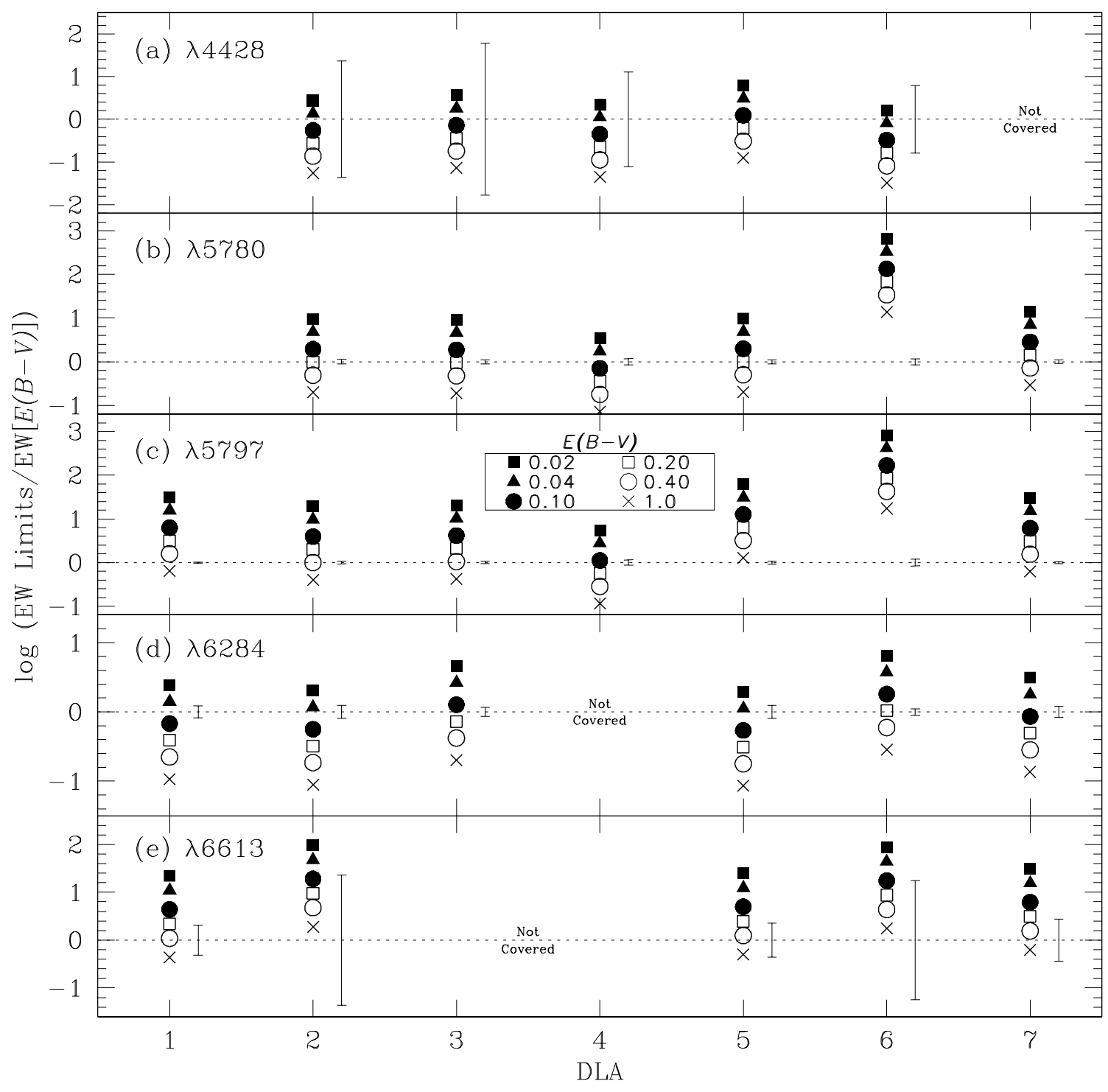

Fig. 10. - The logarithm ratio of the measured equivalent width limits to the equivalent width predicted for multiple values of reddening for each DLA. — (a) $\lambda 4428$ DIB. - (b) $\lambda 5780$ DIB. - $(c)$ $\lambda 5797$ DIB. $-(d) \lambda 6284$ DIB. $-(e) \lambda 6613$ DIB. The equivalent widths of the $\lambda 5780, \lambda 5797$, and $\lambda 6284$ DIBs, for each reddening, were computed using the best-fit Galactic lines from Welty et al. (2006). The equivalent widths of the $\lambda 4428$ and $\lambda 6613$ DIBs, for each reddening, were computed from the relations in Eqs. 4 and 5 (unpublished, T.P. Snow, private communication). The DLAs labeled are as follows: (1) AO 0235+164, (2) Q0738+313, $z=0.091$, (3) Q0738+313, $z=0.221$, (4) B2 0827+243, (5) PKS 0952+179, (6) PKS 1127-145, (7) Q1229-020. We leave the panels of the $\lambda 4428$ and $\lambda 5780$ DIBs for AO $0235+164$ blank because they are detections (Junkkarinen et al. 2004; York et al. 2006) with known reddening, $E(B-V)=0.23$ (Junkkarinen et al. 2004). The zero point (horizontal dotted line) on each plot marks the estimated maximum reddening of the DLA determined from the measured equivalent width limit, or $E(B-V)_{\lim }$ (see Table 4 4 ). The vertical error bars are the $1 \sigma$ errors for the $E(B-V)_{\lim }$ based on the equivalent width limit 


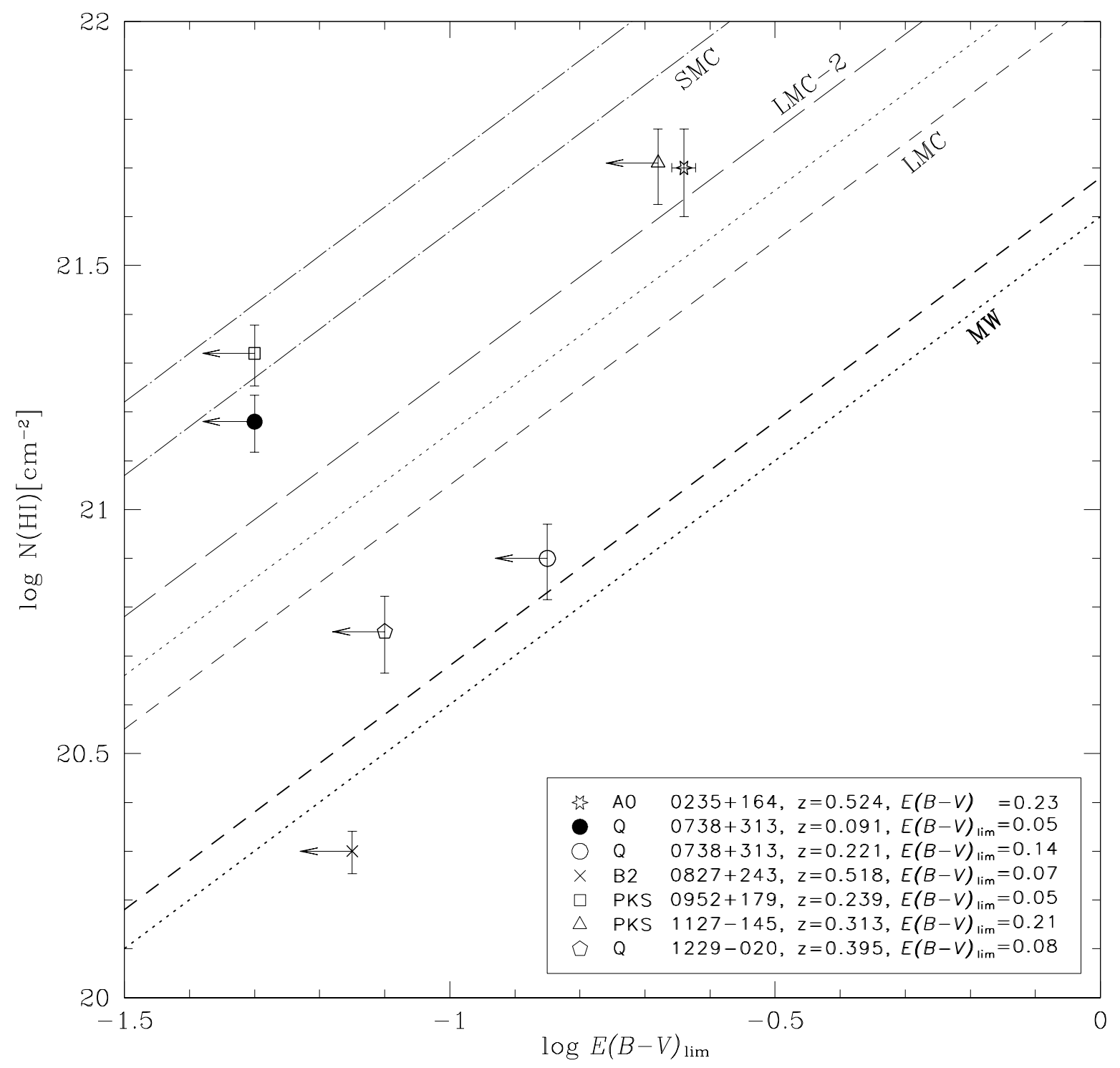

Fig. 11. - The adopted upper limits on the gas-to-dust ratios of the DLAs in our sample. For comparison, the various lines provide ranges of the measured values (given in the text) for the Milky Way (MW), Large Magellanic Cloud (LMC), and Small Magellanic Cloud (SMC). The limits are illustrated with solid leftward arrows, based upon the $\lambda 5780$ and $\lambda 6284$ DIBs. The A0 $0235+164$ reddening measurement (star datum point) is from Junkkarinen et al. (2004). 